San Jose State University

SJSU ScholarWorks

Master's Theses

Master's Theses and Graduate Research

1990

\title{
The synthesis and characterization of bonded liquid crystal stationary phases for use in high performance liquid chromatography
}

Mark Allen Vidensek

San Jose State University

Follow this and additional works at: https://scholarworks.sjsu.edu/etd_theses

\section{Recommended Citation}

Vidensek, Mark Allen, "The synthesis and characterization of bonded liquid crystal stationary phases for use in high performance liquid chromatography" (1990). Master's Theses. 30.

DOI: https://doi.org/10.31979/etd.jt56-dwc8

https://scholarworks.sjsu.edu/etd_theses/30

This Thesis is brought to you for free and open access by the Master's Theses and Graduate Research at SJSU ScholarWorks. It has been accepted for inclusion in Master's Theses by an authorized administrator of SJSU ScholarWorks. For more information, please contact scholarworks@sjsu.edu. 


\section{INFORMATION TO USERS}

The most advanced technology has been used to photograph and reproduce this manuscript from the microfilm master. UMI films the text directly from the original or copy submitted. Thus, some thesis and dissertation copies are in typewriter face, while others may be from any type of computer printer.

The quality of this reproduction is dependent upon the quality of the copy submitted. Broken or indistinct print, colored or poor quality illustrations and photographs, print bleedthrough, substandard margins, and improper alignment can adversely affect reproduction.

In the unlikely event that the author did not send UMI a complete manuscript and there are missing pages, these will be noted. Also, if unauthorized copyright material had to be removed, a note will indicate the deletion.

Oversize materials (e.g., maps, drawings, charts) are reproduced by sectioning the original, beginning at the upper left-hand corner and continuing from left to right in equal sections with small overlaps. Each original is also photographed in one exposure and is included in reduced form at the back of the book.

Photographs included in the original manuscript have been reproduced xerographically in this copy. Higher quality 6" x 9" black and white photographic prints are available for any photographs or illustrations appearing in this copy for an additional charge. Contact UMI directly to order.

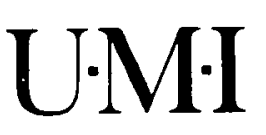



The synthesis and characterization of bonded liquid crystal stationary phases for use in high performance liquid chromatography

Vidensek, Mark Allen, M.S.

San Jose State University, 1990 



\title{
THE SYNTHESIS AND CHARACTERIZATION OF BONDED IIQUID CRYSTAL STATIONARY PHASES FOR USE IN HIGH PERFORMANCE IIQUID CHROMATOGRAPHY
}

\author{
A Thesis \\ Presented to \\ The Faculty of the Department of Chemistry \\ San Jose State University \\ In Partial Fulfillment \\ of the Requirements for the Degree \\ Master of Science
}

By

Mark Allen Vidensek

August, 1990 
APPROVED FOR THE DEPARTMENT OF CHEMISTRY

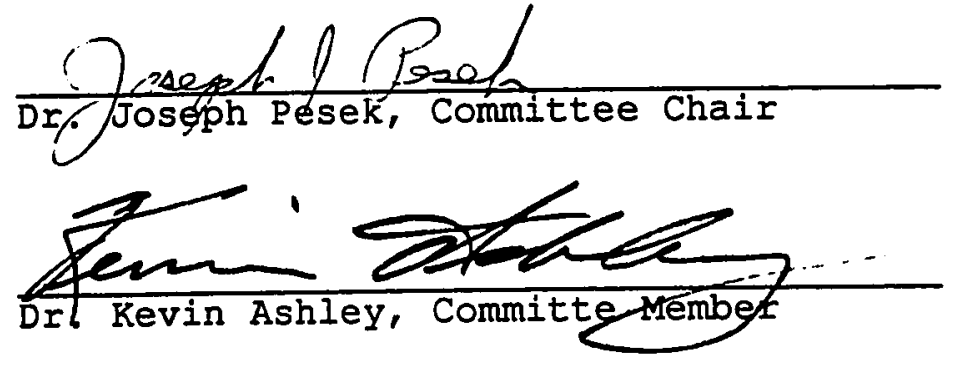

Bradley M. SEr ne

APPROVED FOR THE UNIVERSITY

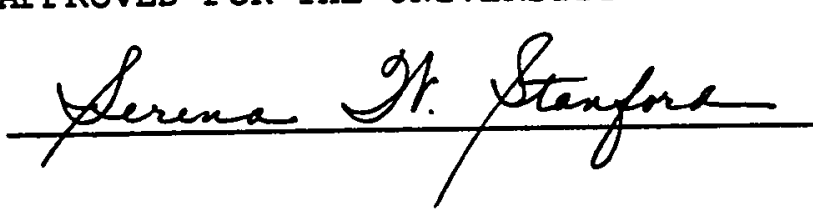


TABLE OF CONTENTS

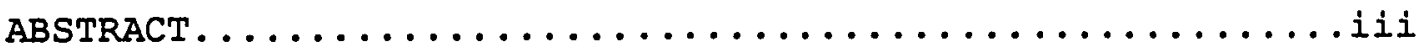

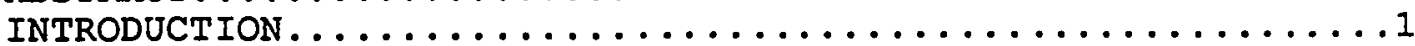

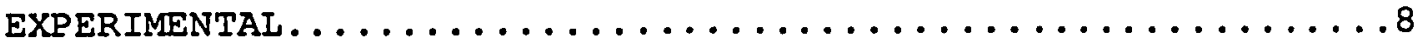

Synthesis of

[4-(Allyloxy) benzoyl] biphenyl................

4- [ \{4-(Allyloxy) benzoyl\}oxy $]-4$ '-methoxy biphenyl.....10

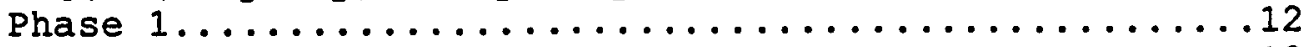

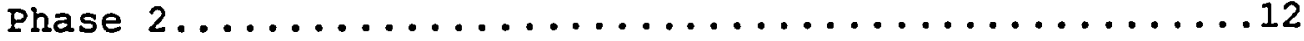

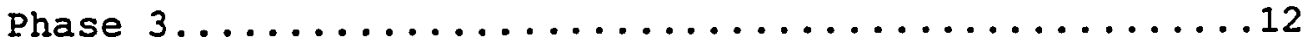

Synthesis of silane reagent of

[4-(Allyloxy) benzoyl] biphenyl...............12

$4-[\{4-($ Allyloxy) benzoyl\}oxy $]-4$ '-methoxy biphenyl.....12

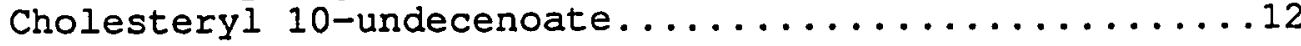

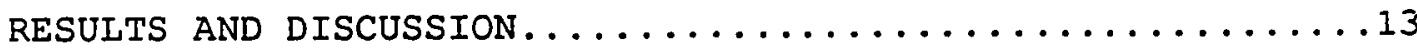

Spectral Analysis of

FTIR of $[4-$ (Allyloxy) benzoyl] biphenyl..........13

H-NMR of $[4-($ Allyloxy) benzoyl] biphenyl...........

FTIR of [4-(Allyloxy) benzoyl] biphenyl silane

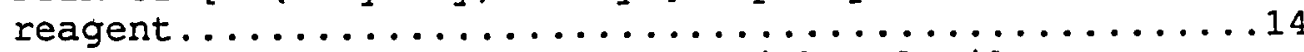

H-NMR of [4-(Allyloxy) benzoyl] biphenyl silane

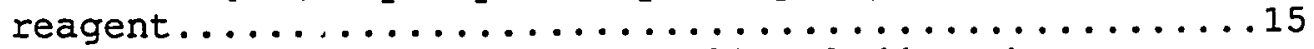

FTIR of $4-[\{4-($ Allyloxy) benzoyl\}oxy $]-4$ '-methoxy

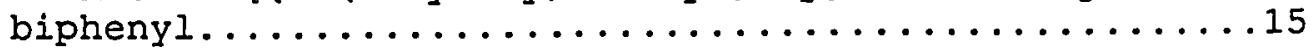

H-NMR of $4-[(4-($ Allyloxy $)$ benzoyl $\}$ oxy $]-4$ '-methoxy

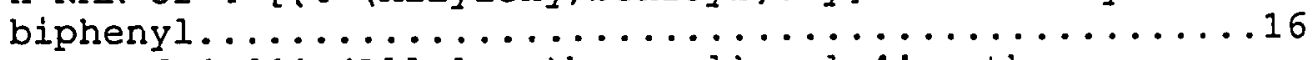

FTIR of 4-[\{4-(Allyloxy)benzoyl\}oxy]-4'-methoxy

biphenyl silane reagent...................

H-NMR of 4- [\{4-(Allyloxy) benzoyl\}oxy]-4'-methoxy

biphenyl silane reagent.................. $\ldots \ldots$

FTIR of Cholesteryl 10-undecenoate..............18

H-NMR of Cholesteryl 10-undecenoate.............18

FTIR of Cholesteryl 10-undecenoate silane reagent....19

H-NMR of Cholesteryl 10-undecenoate silane reagent....19

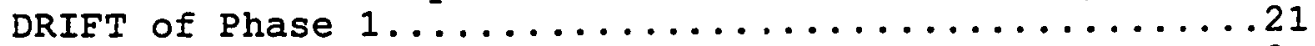

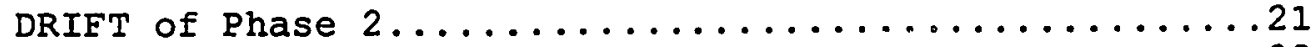

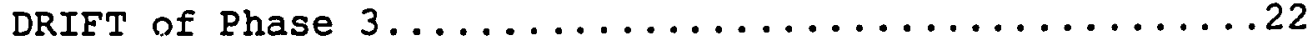

Elemental analysis of

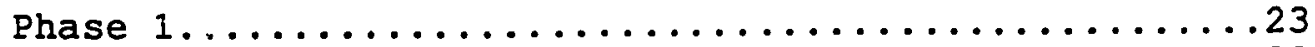

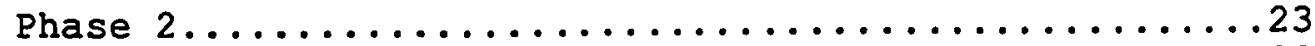

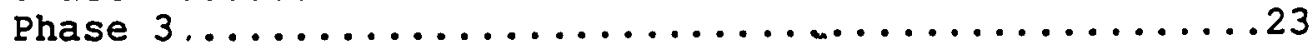


Thermal analysis of

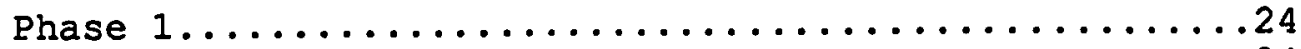

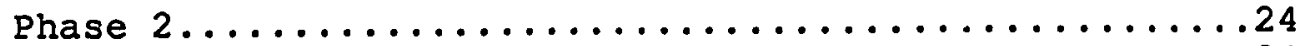

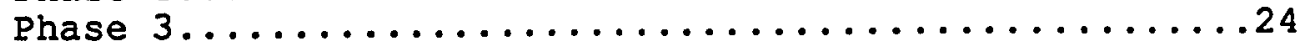

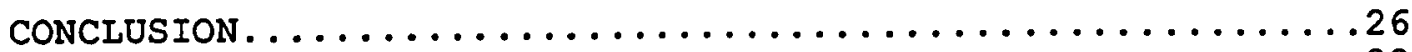

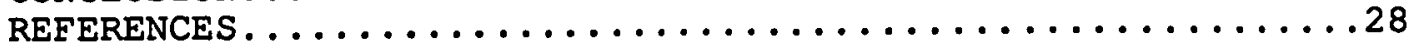

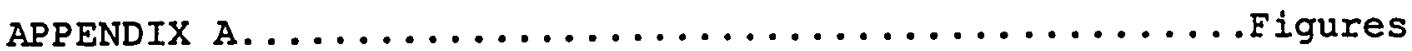


ABSTRACT

THE SYNTHESIS AND CHARACTERIZATION OF BONDED LIQUID CRYSTAL STATIONARY PHASES FOR USE IN HIGH PERFORMANCE LIQUID CHROMATOGRAPHY

By Mark Allen Vidensek

Two bonded nematic liquid crystal stationary phases, for use in HPLC, were prepared by bonding the silanized forms of [4-(Allyloxy)benzoyl] biphenyl and 4-[\{4(Allyloxy) benzoyl\} oxy]-4'-methoxy biphenyl to the surface of silica (Phases 1 and 2, respectively). An attempt was also made to prepare a bonded cholesteric liquid crystal stationary phase, for use in HPLC, by bonding the silanized form of Cholesteryl 10-undecenoate to the surface of silica (Phase 3). However, FTIR and H-NMR spectral studies of the silanization reaction products revealed that Cholesteryl 10undecenoate was not successfully silanized. Phases 1 and 2 were characterized by DRIFT, elemental and thermal (DSC) analyses. The DRIFT spectra of Phases 1 and 2 suggest that a significant amount of organic moiety had been successfully bonded to silica. The carbon analysis revealed a surface coverage of 3.55 and $4.74 \mathrm{umol} / \mathrm{M}^{2}$ for Phases 1 and 2, respectively. The forward DSC traces of Phases 1 and 2 suggest that a broad endothermic phase transition occurred within the temperature range of 300 to $425^{\circ} \mathrm{C}$. 


\section{INTRODUCTION}

In the late 1960 's, high performance liquid chromatography (HPLC) emerged as a qualitative and quantitative analytical method [1]. In the past 25 years, HPIC instruments have changed considerably and today are quite sophisticated. The advent and easy access to extensive computer memories may have changed forever the way in which chemists acquire and manipulate liquid chromatograms, but it is still within the realm of the column that chromatographic separations occur. It is for this reason that the search for a novel and multipurpose stationary phase is of keen interest to liquid chromatographers.

The stationary phase in HPLC can be either solid or liquid. If the stationary phase is solid, the solute molecules compete for a limited number of adsorption sites on the surface of the solid, for example silica gel. The surface of silica gel is covered with polar silanol groups, $-\mathrm{Si}-\mathrm{OH}$, which interact strongly with polar solute molecules. This interaction decreases with decreasing solute polarity. Therefore, retention is based on selective adsorption. Expectedly, poor resolution results when the solute molecules are nonpolar since they are partitioned mainly in the mobile phase, or eluent. If the stationary phase is a liquid, it must be immobilized on a solid support.

Iiquid stationary phases can be either coated onto or chemically bonded to the solid support. In a conventionally 
coated column, this liquid is physically adsorbed onto the silica. The resulting silica particle often has a multimolecular layered coating. However, this leads to low mass transfer which reduces column efficiency by increasing the height equivalent of a theoretical plate (HETP), and consequently chromatographic band broadening results [2] . Also, column bleed can be a serious problem since these physically adsorbed liquids are solublized by the eluent. These problems have been significantly eliminated in bonded phase chromatography (BPC) by chemically bonding an organic moiety to the solid support resulting in a bonded brush. It should be noted, however, that excessively long brushes are capable of folding back upon themselves; this reduces column efficiency by increasing the HETP. Shorter brushes are desirable.

These bonded phases were prepared by reaction between the free silanol groups on the silica surface and alcohols. However, the resulting $-\mathrm{Si}-\mathrm{O}-\mathrm{C}$ - bond system was found to be unstable towards extremes of $\mathrm{pH}$ and temperatures $[3,4]$. In addition, only a limited number of these bonded phases can be readily prepared. This futher limited their use. New reaction schemes were necessarily developed.

One approach developed involves the chlorination of silica by reaction with thionyl chloride, see Eq. 1. The brush is then bonded to the chlorinated surface via a 


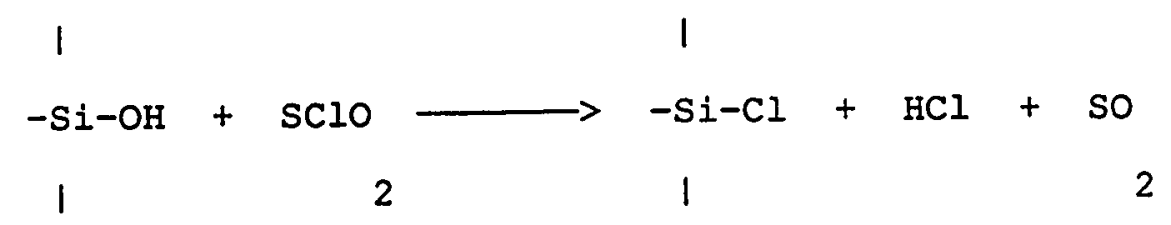

Grignard reaction, see Eq. 2, [5].

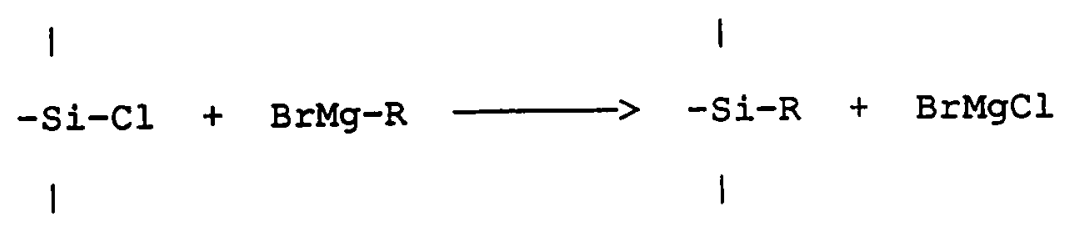

The resulting -Si-O-Si-C- bond has greater thermal stability and is less susceptible to extremes of $\mathrm{pH}$ [3]. However, the alkyl group of the Grignard reagent is limited to those species which do not contain active groups like $-\mathrm{CN}, \mathrm{C}=0$, acidic protons, etc; these groups are known to reduce Grignard reagents. A second approach avoids this limiting aspect of Grignard reagents completely.

In an alternative method, an organic species containing a terminal $\mathrm{C}=\mathrm{C}$ bond is chlorinated via a hydrosilanization addition reaction, see Eq. 3, [6].

$$
\begin{array}{rrcr}
\mathrm{R}-\mathrm{CH}=\mathrm{CH} & +\mathrm{HClSi}(\mathrm{CH}) & \longrightarrow \mathrm{R}-\mathrm{CH}-\mathrm{CH}-\mathrm{SiCl}(\mathrm{CH}) \\
2 & 32 & 22 & 32
\end{array}
$$

The product is then bonded to the silica surface, see Eq. 4. 


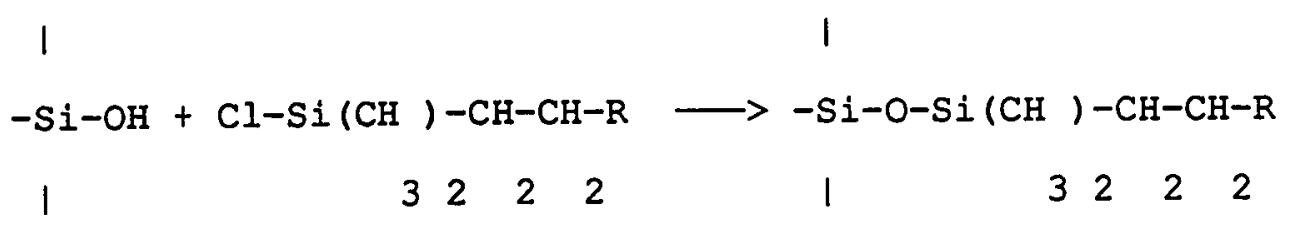

This method has greater versatility than the Grignard reagent approach. In addition, the stable -Si-O-Si-C- bond system is maintained in the product. Equation 4 outlines the most commonly used method for modifying the surface of silica. Equations 3 and 4 suggest that one can selectively generate a bonded brush with specific retention characteristics by simply choosing the proper organic starting material, $-R$. A class of organic species of special interest in BPC today are liquid crystals. Most organic substances display a sharp transition during the melting process. Materials that do not exhibit this type of transition possess mesophases; a mesophase is intermediate to the solid and isotropic liquid states. Mesomorphic states that contain a certain degree of order are referred to as liquid crystals [7].

Iiquid crystals can be divided into two categories: thermotropic and lyotropic. Lyotropic liquid crystals are formed by mixing two, or more, compounds together, that is, a solute and solvent. It is the interaction between these species which is responsible for the order of the mesomorphic state. The molecules of lyotropic liquid cystals are long and rod-like in shape with axial ratios, 
length:width, not greater than 15. This type of liquid crystal, however, is unstable towards thermal changes [7]. Thermotropic liquid crystals are produced by heating the pure solid. The resulting liquid crystallinity often exists over a range of several degrees, some spanning room temperature. The molecules of thermotropic liquid crystals are also rod-like in shape but with axial ratios of only 4 to 5 . Their conformations contain a central linkage flanked on both sides by terminal groups; the central linkage and terminal groups are generally separated by aromatic rings [8].

There are three classes of thermotropic liquid crystals: smectic, nematic and cholesteric. These classes have been characterized by the degree of their liquid crystalline order. Smectic liquid crystals possess the highest degree of order, being comprised of molecules whose long molecular axes run parallel. These axes lie normal, or tilted, relative to the layers of the liquid crystal, see figures $1 \mathrm{a}$ and $1 \mathrm{~b}$. Cholesteric liquid crystals are similar to the smectic order except that their molecular axes lie within the planes of the layers of the liquid crystal, see figure 1c. Liquid crystals of the nematic order do not form layers, but their long molecular axes do run parallel, see figure 1d. Nematic liquid crystals are, of course, the least ordered [7].

The lower members of a homologous series of 
thermotropic materials are generally nematic, while the smectic order predominates as the series increases; however, the length of these molecules are considerably less than those of lyotropic liquid crystals.

In BPC, brush size, as pointed out earlier, is a critical factor in choosing a material for bonding to silica. Clearly, a bonded lyotropic liquid crystal would exhibit reduced mass transfer because of the resulting multi-molecular layered coating on the silica particle, similar to that of a conventionally coated column. A bonded nematic liquid crystal, on the other hand, would ensure a mono-molecular coating of the silica since back folding is less likely with shorter brushes. Therefore, a nematic liquid crystal bonded to silica should produce the most efficient HPLC stationary phase of a liquid crystal type. A bonded nematic phase should also selectively retain those solute molecules which are rod like in shape. Selectivity of this nature has been shown to exist by Laub and coworkers [9].

In our present study, we attempted to synthesize and characterize three bonded liquid crystal stationary phases for use in HPLC. Phases 1 and 2 were generated from [4(Allyloxy)benzoyl] biphenyl, see figure $2 a$, and the nematic liquid crystal 4-[\{4-(Allyloxy) benzoyl\}oxy]-4'-methoxy biphenyl, see figure 2b, respectively. Phase 3 was prepared from the cholesteric liquid crystal Cholesteryl 10- 
undecenoate, see figure $2 c$. The terminal $C=C$ bond of these species were silanized by reaction with dimethylchlorosilane in the presence of hexachloroplatinic acid, see Eq. 3. The resulting chlorinated species, silane reagents, were then chemically bonded to the surface of silica, see Eq. 4. Infrared (FTIR) and H-1 NMR spectra of [4-(Allyloxy) benzoyl] biphenyl, 4-[\{4-(Allyloxy) bezoyl\}oxy]-4'-methoxy biphenyl and Cholesteryl 10-undecenoate were taken. This was done to ensure that the synthesis of the biphenyls had been successful. These spectra were also compared to those taken of the hydrolyzed silane reagents. The success of the silanization addition reactions were determined by comparing the relative magnitudes of the aliphatic and olefinic signals from these spectra, see Eq. 3 .

Instrumental, elemental and thermal analysis methods were employed to characterize the three bonded stationary phases. A diffuse reflectance infrared fourier transform (DRIFT spectrum) of bare silica was taken and compared to DRIET spectra of the bonded phases in an attempt to determine the extent of reaction by comparing the relative magnitudes of the silanol's $\mathrm{O}-\mathrm{H}$ stretching bands, see Eq. 4. In addition, elemental analyses were performed on the bonded phases in an attempt to determine the extent of surface coverage. The three bonded stationary phases were also thermally analyzed by differential scanning calorimetry (DSC) in an attempt to ascertain the thermodynamic nature of 
their mesomorphic transitions.

EXPERIMENTAI

Materials: All chemicals used in this study were of reagent grade. All liquid reagents and solvents employed in moisture sensitive reactions were distilled and collected over type 4A molecular sieves. All solid materials employed in moisture sensitive reactions were dried at $110^{\circ} \mathrm{C}$ for 24 hours prior to use. All dried solids were cooled to $25^{\circ} \mathrm{C}$ in a vacuum descicator over drierite. The Cholestery $10-$ undecenoate was dried in a vacuum descicator over drierite for 24 hours prior to use. The Cholestery 10 -undecenoate was purchased from Chemical Dynamics Corporation (South Plainfield, New Jersey). Nucleosil 300-10, with a particle size of 10 um and an average pore diameter of $300 \dot{A}$, was used as the solid support. The silica was purchased from Alltech Associates, Inc. (Deerfield, Illinois). The KBr used in the infrared studies was of spectral grade and dried for a minimum of one hour at $110^{\circ} \mathrm{C}$ prior to use. The solvent used in the H-NMR studies was deuterated chloroform; the solvent was labelled $99.8 \%$ deuterated.

Apparatus: All glassware used in moisture sensitive synthetic work was dried at $110^{\circ} \mathrm{C}$ for 24 hours prior to use and assembled hot with a nitrogen purge. The infrared spectral studies were performed on a Perkin-Elmer model 1800 spectrometer. The H-1 NMR spectral studies were performed on a Varian EM 390 spectrophotometer. The elemental analyses 
were performed at U.C. Berkeley Micro Lab (Berkeley, Ca.). The DSC studies were run on a Perkin-Elmer Series 7 Thermal Analysis System.

Procedure: [The following procedure can be further referenced in [9].]

Synthesis of 4-(Allyloxy)benzoic acid: To a $1000 \mathrm{ml}$ three neck round bottom flask equipped with a $60 \mathrm{~cm}$ reflux condenser, a $250 \mathrm{ml}$ self-equalizing addition funnel, a magnetic stirring bar and an oil bath, was added $87.95 \mathrm{~g}$ of 4-Hydroxy benzoic acid; to this was added, at $25^{\circ} \mathrm{C}, 400 \mathrm{ml}$ of methanol with stirring; a brownish solution developed. To this solution was added dropwise $105 \mathrm{~g}$ of $\mathrm{KOH}$ dissolved in $125 \mathrm{ml}$ DI water. This was heated to reflux and $90.85 \mathrm{~g}$ of allylbromide was then added over a one hour period. The solution was refluxed for eight hours. Next, $250 \mathrm{ml}$ of methanol was distilled out. The reaction mixture was cooled to $25^{\circ} \mathrm{C}$, and was added to $100 \mathrm{ml}$ of $\mathrm{DI}$ water. The aqueous solution was extracted three times with $200 \mathrm{ml}$ diethyl ether; the ether layer was discarded. The solution was heated to $40^{\circ} \mathrm{C}$ and $300 \mathrm{ml}$ of $20 \% \mathrm{HCl}$ was added. The crude acid was recovered by vacuum filtration and recrystalized seven times from ethanol yielding $43.53 \mathrm{~g}(25 \%), M P 167^{\circ} \mathrm{C}$. Synthesis of 4-(Allyloxy)benzoyl chloride: To a two neck $100 \mathrm{ml}$ round bottom flask equipped with a $50 \mathrm{~cm}$ reflux condenser, a Teflon septum with a nitrogen line, a magnetic stirring bar and an oil bath, was added $25 \mathrm{ml}$ of thionyl 
chloride to $25.40 \mathrm{~g}$ of the 4-(Allyloxy)benzoic acid prepared above. Next, 10 drops of Dimethylformamide was added to the solution. The reaction mixture was protected from moisture and stirred over night under nitrogen. The mixture was then heat to $60^{\circ} \mathrm{C}$ for two hours with stirring. The residual thionyl chloride was removed by vacuum. The crude product was vacuum distilled at $1 \mathrm{~mm} \mathrm{Hg} / 100^{\circ} \mathrm{C}$ yielding $11.50 \mathrm{~g}$ (41\%) of a pale yellow liquid, which was stored in a tightly sealed bottle under nitrogen.

Synthesis of [4-(Allyloxy)benzoyl] biphenyl: To a two neck $250 \mathrm{ml}$ round bottom flask equipped with a $50 \mathrm{~cm}$ reflux condenser, a Teflon septum with a nitrogen line, a magnetic stirring bar and an oil bath, was added $4.93 \mathrm{~g}$ of 4phenylphenol dissolved in $50 \mathrm{ml}$ of dry pyridine under nitrogen. Next, $6.13 \mathrm{~g}$ of 4-(Allyloxy)benzoyl chloride was added with stirring. The mixture was stirred for three hours at $25^{\circ} \mathrm{C}$ and was then heated to $60^{\circ} \mathrm{C}$ for an additional two hours. After the reaction mixture had cooled to $25^{\circ} \mathrm{C}$, it was added to $200 \mathrm{ml}$ of DI water and acidified with $20 \% \mathrm{HCl}$. The resulting precipitate was collected by vacuum filtration and washed with $200 \mathrm{ml}$ cold DI water. The crude product was recrystallized from a $1: 1 \mathrm{v} / \mathrm{v}$ acetone:ethanol mixture yielding $8.45 \mathrm{~g}\left(88^{\circ}\right)$, MP $138-140^{\circ} \mathrm{C}$.

Synthesis of 4-[\{4-(AIIyloxy)benzoyl\}oxy $]-4$ '-methoxybiphenyl: To a three neck $1000 \mathrm{ml}$ round bottom flask equipped with a self-equalizing addition funnel, a $50 \mathrm{~cm}$ 
refiux condenser, a Teflon septum with a nitrogen line, a magnetic stirring bar and an ice bath, was added $400 \mathrm{ml}$ of $10 \%$ aqueous $\mathrm{NaOH}$ cooled to $0^{\circ} \mathrm{C}$. Next, $75.50 \mathrm{~g}$ of $4,4^{\prime}-$ dihydroxybiphenyl was added with vigorous stirring. To this was added $48.64 \mathrm{~g}$ of dimethyisulfate over a one hour period with vigorous stirring. After the addition was complete, the resulting precipitate was recovered by vacuum filtration with a sintered glass filter and was redissolved in $400 \mathrm{ml}$ of $10 \%$ aqueous $\mathrm{NaOH}$. The solution was then heated briefly to boiling and was allowed to cool for filtration. The solid mass was then placed in $200 \mathrm{ml}$ of water and was heated to boiling and filtered while hot. The filtrate was then heated to $70^{\circ} \mathrm{C}$ and acidified with $20 \% \mathrm{HCL}$, causing crude 4hydroxy-4'-methoxybiphenyl to precipitate which was recrystallized twice from ethanol.

To a three neck $250 \mathrm{ml}$ round bottom flask equipped with a $100 \mathrm{ml}$ self-equalizing addition funnel, a $50 \mathrm{~cm}$ reflux condenser, a Teflon septum with a nitrogen line, a magnetic stirring bar and an oil bath, was added $3.62 \mathrm{~g}$ of 4-hydroxy4'-methoxybiphenyl; the 4-hydroxy-4'-methoxybiphenyl was dissolved in $100 \mathrm{ml}$ of dry pyridine under a nitrogen blanket. Next, $4.19 \mathrm{~g}$ of 4 -(Allyloxy) benzoyl chloride dissolved in $20 \mathrm{ml}$ of dry pyridine was added over a one hour period with stirring. The reaction mixture was stirred for an additional 3 hours at $25^{\circ} \mathrm{C}$ and was then heated to $60^{\circ} \mathrm{C}$ for an additional 2 hours. The reaction mixture was cooled 
to $25^{\circ} \mathrm{C}$ and added to $400 \mathrm{mI}$ DI water. The solution was acidified with $20 \% \mathrm{HCl}$. The precipitate was recovered by vacuum filtration and was washed with $400 \mathrm{ml}$ of saturated sodium bicarbonate and then with $400 \mathrm{ml}$ DI water. The crude product was recrystallized twice from acetone yielding 4.27 g $(84 \%), M P 147-249^{\circ} \mathrm{C}$.

Silanization and silica bonding of the silane reagents: [The following procedure can be further referenced in [6].] To a two neck $100 \mathrm{ml}$ round bottom flask equipped with a $50 \mathrm{~cm}$ reflux condenser, a Teflon septum with a nitrogen line, a magnetic stirring bar and an oil bath, was added $11.8 \mathrm{mmol}$ of the organic moiety dissolved in $20 \mathrm{mI}$ of dry toluene. Next, $11.8 \mathrm{mmol}$ of dimethylchlorosilane was added to the reaction flask while stirring and purging wtih nitrogen. After the reaction mixture was stirred for 5 minutes, $8 \mathrm{mg}$ of hexachloroplatinic acid was added. The reaction mixture was then heated to $65^{\circ} \mathrm{C}$ and stirred for 10 days under nitrogen, see Eq. 3. Next, $20 \mathrm{ml}$ of freshly distilled toluene was added followed by $6.5 \mathrm{~g}$ of silica and $0.5 \mathrm{ml}$ of dry pyridine. The reaction mixture was stirred for 10 days at $40^{\circ} \mathrm{C}$ under nitrogen, see Eq. 4. The three bonded phases were then washed with $60 \mathrm{ml}$ of toluene followed by a $60 \mathrm{ml}$ wash of ethanol. This wash procedure was repeated six times. 


\section{RESULTS AND DISCUSSION}

INFRARED AND H-1 NMR SPECTRAL ANALYSIS

\section{[4-(Allyloxy)benzoyl] biphenyl}

The infrared spectrum of [4-(Allyloxy)benzoyl] biphenyl is shown in Figure 3. The infrared band appearing at 3072 cm- I is due to the $\mathrm{C}-\mathrm{H}$ stretching frequency of a terminal alkene. Also, a band at $1647 \mathrm{~cm}-1$ futher supports the presence of a $\mathrm{C}=\mathrm{C}$ bond; this is due to a nonconjugated $\mathrm{C}=\mathrm{C}$ group. The cluster of bands in the region of $3072-3017 \mathrm{~cm}-1$ arise from the $\mathrm{C}-\mathrm{H}$ stretching frequencies of an aromatic ring system. Also, the presence of aromatic character is further evident from those bands appearing at 1608 and 1511 $\mathrm{cm}-1$. The infrared bands in the region of $2980-2893 \mathrm{~cm}-1$ correspond to aliphatic $\mathrm{C}-\mathrm{H}$ stretching; note the weak nature of these bands. The strong band appearing at $1731 \mathrm{~cm}-1$ is characteristic of an ester $C=0$ stretching frequency; in addition, the two bands appearing at 1260 and $1169 \mathrm{~cm}-1$ are indicative of an ester. The peaks at 1214 and $1070 \mathrm{~cm}-1$ correspond to the $\mathrm{Ar}-\mathrm{O}$ and Ar-O-C bond systems, respectively. The peak assignments discussed above are consistant with the structure of [4-(Allyloxy) benzoyl] biphenyl shown in figure $2 a$.

The H-1 NMR spectrum of [4-(Allyloxy)benzoyl] biphenyl is shown in figure 4. The multiplet signal in the region of $7.50 \mathrm{ppm}$ corresponds to aromatic protons, Ar-B. The multiplet at $6.10 \mathrm{ppm}$ is due to a single vinyl proton with 
four neighboring protons, $\mathrm{H} 2 \mathrm{C}=\mathrm{CH}-\mathrm{CH} 2-$; the complex splitting pattern is due to the vinyl protons experiencing different chemical environments. A similar effect is observed at 5.45 ppm which arises from two vinyl protons on the same carbon but experiencing different chemical environments due to an alkyl group being cis or/and trans, $\mathrm{B2C}=\mathrm{CH}-$; as a result of this differing environment, a doublet is not observed. The doublet at $4.75 \mathrm{ppm}$ corresponds to two methylene protons with one neighboring proton, $=\mathrm{CH}-\mathrm{CH} 2-\mathrm{O}-$. The singlet at 1.80 ppm is due to water in the solvent. The H-1 NMR peak assignments discussed above were substantiated by integration data analysis and are consistant with the structure of [4-(Allyloxy)benzoyl] biphenyl shown in figure $2 a$.

The analysis of infrared and $H-1$ NMR spectra suggest quite strongly that the synthesis of [4-(Allyloxy)benzoyl] biphenyl was successful. Silanized [4-(Allyloxy)benzoyl] biphenyl

The infrared spectrum of the silanized [4-(Allyloxy) benzoyl] biphenyl is shown in figure 5, see Eq. 3. The intensity of the aliphatic $\mathrm{C}-\mathrm{H}$ stretching band at 3017-2893 cm-1 has increased significantly relative to the same band shown in figure 3 . This suggests greater $\mathrm{C}-\mathrm{H}$ character. Also, the absence of the $\mathrm{C}-\mathrm{H}$ stretching frequency bands at 3072 and $1647 \mathrm{~cm}-1$ in figure 4 implies that the terminal $\mathrm{C}=\mathrm{C}$ is no longer present in the silanization reation product. 
The H-1 NMR spectrum of silanized [4-(Allyloxy)benzoyl] biphenyl is shown in figure 6, see Eq. 3. By comparing figures 4 and 6 , we can see that those peaks associated with the $\mathrm{C}=\mathrm{C}$ bond are absent from the spectra of silanized [4(Allyloxy)benzoyl] biphenyl, figure 6 . These peaks have been replaced by a series of H-NMR signals appearing at 4.00, 1.80 and $0.70 \mathrm{ppm}$, see figure 6 . These signals correspond to the methylene protons of the silane reagent; the peak appearing at 1.25 corresponds to water in the solvent. In addition, a peak appearing at $0.25 \mathrm{ppm}$ in figure 6 is due to the methyl protons of the silane reagent, similar to TMS. This implies that the $\mathrm{C}=\mathrm{C}$ bond has been silanized. The analysis of infrared and $H-1$ NMR spectra suggest quite strongly that the silanization of [4-(Allyloxy)benzoyl] biphenyl was successful. $4-[\{4-($ Allyloxy) benzoyl $\}$ oxy $]-4$ '-methoxybipheny 1

The infrared spectrum of $4-[\{4-($ Allyloxy $)$ benzoyl\}oxy $]-$ 4'-methoxybiphenyl is shown in figure 7. The infrared bands in the region of $3080 \mathrm{~cm}-1$ correspond to the $\mathrm{C}-\mathrm{H}$ stretching frequency of a terminal alkene. In addition, a band at 1651 cm-1 further supports the presence of a $\mathrm{C}=\mathrm{C}$ bond; this is due to a nonconjugated $\mathrm{C}=\mathrm{C}$ group. The cluster of bands in the region of $3075-3000 \mathrm{~cm}-1$ correspond to the $\mathrm{C}-\mathrm{H}$ stretching frequency of an aromatic ring system. The presence of aromatic character is further evident from those bands appearing at 1606 and $1509 \mathrm{~cm}-1$. The infrared bands 
appearing in the region of $2980-2900 \mathrm{~cm}-1$ are due to aliphatic $\mathrm{C}-\mathrm{H}$ stretching; note the weak nature of these bands. The strong band appearing at $1733 \mathrm{~cm}-1$ corresponds to the stretching frequency of an ester $C=0$ group; in addition, the bands at 1256 and 1167 are indicative of an ester. The observed peak at $1212 \mathrm{~cm}-1$ is due to the Ar-O-C functional group of the terminal methoxy. The infrared peak assignments discussed above are consistent with the structure of 4-[\{4-(Allyloxy) benzoyl\}oxy]-4'-methoxy biphenyl shown in figure $2 \mathrm{~b}$.

The H-1 NMR spectrum of 4-[\{4-(Allyloxy) benzoyl\}oxy $]-$ 4'-methoxybiphenyl is shown in figure 8. The multiplet signal in the region of $7.50 \mathrm{ppm}$ correspond to aromatic protons, Ar-H. The mulitplet at $6.10 \mathrm{ppm}$ is due to a single vinyl proton with four neighboring protons, $\mathrm{H} 2 \mathrm{C}=\mathrm{CH}-\mathrm{CH} 2-$; the complex spitting pattern is due to the vinyl protons experiencing different chemical environments. A similar effect is observed at $5.45 \mathrm{ppm}$ which arises from two vinyl protons on the same carbon but experiencing different chemical environments due to an alkyl group being cis or/and trans, $\mathrm{B2C}=\mathrm{CH}-;$ as a result of this differing environment, a doublet is not observed. The doublet at $4.65 \mathrm{ppm}$ corresponds to two methylene protons with one neighboring proton, $=\mathrm{CH}-\mathrm{CH} 2-0$. The singlet at $3.80 \mathrm{ppm}$ is due to the methyl protons of the terminal methoxy group, Ar-O-CB3. The H-1 NMR peak assignments discussed above were substantiated by 
integration data analysis and are consistent with the structure of 4-[\{4-(Allyloxy) benzoyl\}oxy $]-4{ }^{\prime}-$ methoxy biphenyl shown in figure $2 \mathrm{~b}$.

The analysis of infrared and $H-1$ NMR spectra suggests quite strongly that the synthesis of $4-[\{4-(A l l y l o x y)$ benzoyl $\}$ oxy]-4'-methoxy biphenyl was successful.

Silanized 4-[\{4-(Allyloxy) benzoyl\}oxy]-4'-methoxy biphenyl

The infrared spectrum of the silanized 4-[\{4-(Allyloxy) benzoylfoxy]-4'-methoxy biphenyl is shown in figure 9, see Eq. 3. The intensity of the aliphatic $\mathrm{C}-\mathrm{H}$ stretching band at 2940-2838 cm-1 has increased significantly relative to the same band shown in figure 7 . This suggests greater $\mathrm{C}-\mathrm{H}$ character. However, the presence, though reduced in intensity, of the $\mathrm{C}-\mathrm{H}$ stretching frequency band at $1650 \mathrm{~cm}-1$ in figure 9, compared to the same band shown in figure 7, implies that the terminal $C=C$ bond has not been completely silanized.

The H-1 NMR spectrum of silanized 4-[\{4-(Allyloxy) benzoyl\}oxy]-4'-methoxy biphenyl is shown in figure 10, see Eq. 3. By comparing figures 8 and 10 , we can see that those peaks associated with the $\mathrm{C}=\mathrm{C}$ bond are still present, though reduced in intensity, in the spectrum of silanized 4-[14(Allyloxy) benzoylfoxy]-4'-methoxy biphenyl, figure 10. This implies the $C=C$ bond has not been completely silanized. The peaks appearing at $4.00,1.85$ and $0.65 \mathrm{ppm}$ in figure 10 correspond to the methylene protons of the silane regent. 
This suggests that the $C=C$ bond has been silanized, if only partially. The signal at $1.05 \mathrm{ppm}$ is due to water in the solvent. In addition, a peak appearing at $0.25 \mathrm{ppm}$ in figure 10 is due to the methyl protons of the silane reagent, similar to TMS. This implies that the $\mathrm{C}=\mathrm{C}$ bond has been silanized, if only partially.

The analysis of infrared and $H-1$ NMR spectra suggest quite strongly that the silanization of 4- (\{4-(Allyloxy) benzoyl\}oxy]-4'-methoxy biphenyl was only partially successful. If the $\mathrm{C}=\mathrm{C}$ bond had been completely silanized, the peaks associated with the $\mathrm{C}=\mathrm{C}$ bond system would not have been observed in figures 9 and 10 .

Cholesteryl 10-undecenoate

As noted earlier, Cholesteryl 10-undecenoate was purchased. Therefore, the following spectral analyses have focused upon determining the extent of silanization of the terminal $\mathrm{C}=\mathrm{C}$ bond, see figure $2 \mathrm{c}$.

The infrared spectrum of Cholesteryl 10-undecenoate is shown in figure 11. The weak band appearing at $3080 \mathrm{~cm}-1$ arises from the $\mathrm{C}-\mathrm{H}$ stretching frequency of a terminal alkene. The strong infrared band in the region of 2926-2852 Cm-1 is due to aliphatic $\mathrm{C}-\mathrm{H}$ stretching.

The H-1 NMR spectrum of Cholesteryl 10-undecenoate is shown in figure 12. The proton signals of greatest interest appear at 5.50 and $4.75 \mathrm{ppm}$. The H-NMR signal appearing at $4.75 \mathrm{ppm}$ corresponds to the terminal vinyl protons, $\mathrm{B} 2 \mathrm{C}=\mathrm{CH}-$. 
The signal appearing at $5.50 \mathrm{ppm}$ is due to the methine proton, $\mathrm{H} 2 \mathrm{C}=\mathrm{CE}-\mathrm{CH} 2-$, of the terminal vinyl group. Silanized Cholesteryl 10-undecenoate

The infrared spectrum of the silanized Cholesteryl 10undecenoate is shown in figure 13, see Eq. 3. The infrared band at $3080 \mathrm{~cm}-1$ corresponds to the $\mathrm{C}-\mathrm{H}$ stretching frequency of a terminal alkene. In addition, the aliphatic stretching frequency band in the region of $2926-2852 \mathrm{~cm}-1$ of figure 13 has not changed significantly relative to the same band shown in figure 11. If the silanization reaction of Colesteryl 10-undecenoate had been successful, these bands would have been altered significantly.

The H-1 NMR spectrum of silanized Cholesteryl 10undecenoate is shown in figure 14, see Eq. 3. By comparing figures 12 and 14, we can see that those peaks associated with the $C=C$ bond system are still present in the spectrum of silanized Cholesteryl 10-undecenoate, figure 14. In addition, there is an absence of any proton signal at 0.25 ppm in figure 14. These data suggest that the $\mathrm{C}=\mathrm{C}$ bond is still present.

The analysis of infrared and $\mathrm{H}-1$ NMR spectra strongly suggest that the silanization of Cholesteryl 10-undecenoate was not successful.

From the above discussions, it would appear that the methods used in this study to silanize the organic moieties may have some limitations. As noted earlier, nematic liquid 
crystals are less ordered than choleresteric liquid crystals. This could explain why Cholesteryl 10-undecenoate was not successfully silanized. Recall from figure lc that the long molecular axes of Cholesteryl 10-undecenoate run parellel and within the planes of the layers of the liquid crystal. If this highly ordered orientation were extended to the solution phase, similar to lyotropic liquid crystals, the terminal $\mathrm{C}=\mathrm{C}$ bond of Cholestery 10 -undecenoate could be effectively shielded from addition reactions. This could partially explain why our efforts to silanize Cholesteryl 10-undecenoate were unsuccessful. It should also be noted that the nematic liquid crystal 4-[(4-(Allyloxy) benzoyl\}oxy] -4'-methoxy biphenyl was only partially silanized while [4(Allyloxy)benzoyl] biphenyl, which is not a liquid crystal, appeared to completely silanized. This suggests that the success of a silanization is inversely related to the degree of order. Further, this would appear to exclude the use of highly ordered smectic liquid crystals. This limitation warrants further study. DRIFT SPECTRAL ANALYSIS

Bare silica

The DRIFT spectrum of bare silica is shown in figure 15. The infrared band of greatest interest appears at 3745 cm-1. This band corresponds to the stretching frequency of the silanol groups, Si-O-H, on the silica surface. The weak band appearing at $2950 \mathrm{~cm}-1$ is due to physically adsorbed 
organic material.

Phase 1

The DRIFT spectrum of phase 1 is shown in figure 16, see Eq. 4. The cluster of infrared bands in the region of $3066 \mathrm{~cm}-1$ are due to the $\mathrm{C}-\mathrm{H}$ stretching frequency of an aromatic ring system. In addition, the two bands appearing at 1607 and $1517 \mathrm{~cm}-1$ further support the presence of aromatic character. The band appearing at $2941 \mathrm{~cm}-1$ arises from aliphatic $\mathrm{C}-\mathrm{H}$ stretching. The band at $1735 \mathrm{~cm}-1$ is due to the stretching frequency of an ester $C=0$ group. Note the absence of the sharp Si-OH stretching frequency band at 3745 $\mathrm{cm}-1$. This implies the $\mathrm{Si}-\mathrm{OH}$ groups are no longer present on the surface of the reacted silica; figure 17 shows a comparison of the $3700 \mathrm{~cm}-1$ region of figures 15 and 16 . This strongly suggests that the silanol groups have been replaced by the [4-(Allyloxy)benzoyl] biphenyl silane reagent resulting in a bonded brush.

Phase 2

The DRIFT spectrum of Phase 2 is shown in figure 18, see Eq. 4. The cluster of bands in the region of $3050 \mathrm{~cm}-1$ correspond to the $\mathrm{C}-\mathrm{H}$ stretching frequency of an aromatic ring system. Also, the two bands appearing at 1607 and 1506 cm-1 further support aromatic character. The infrared band at $2960 \mathrm{~cm}-1$ is due to aliphatic $\mathrm{C}-\mathrm{H}$ stretching. The infrared band appearing at $1733 \mathrm{~cm}-1$ corresponds to the stretching frequency of an ester $\mathrm{C}=0$ group. Note the absence 
of the Si-OH stretching frequency at $3745 \mathrm{~cm}-1$. This implies the Si-OH groups are no longer present on the surface of the reacted silica; figure 19 shows a comparison of the 3700 $\mathrm{cm}^{-1}$ region of figures 15 and 18 . This strongly suggests that the silanol groups have been replaced by the 4 [14(Allyloxy)benzoyl\}oxy]-4'-methoxy biphenyl silane reagent resulting in a bonded brush.

\section{Phase 3}

The DRIFT spectrum of Phase 3 is shown in figure 20, see Eq. 4. As mentioned ealier, the silanization reaction of Cholesteryl 10-undecenoate did not appear to have been successful. The DRIFT spectrum of Phase 3 seems to confirm this postulation. Note the presence of the Si-OH band at $3745 \mathrm{~cm}-1$, though reduced in intensity relative to the same band for bare silica; figure 21 shows a comparison of the $3700 \mathrm{~cm}^{-1}$ region of figures 15 an 20 . The band at $2968 \mathrm{~cm}-1$ is due to aliphatic $\mathrm{C}-\mathrm{H}$ stretching; however, it does not appear to correspond to the aliphatic $\mathrm{C}-\mathrm{H}$ stretching frequency band observed in figure 11. In addition, there are no functionality bands observed which are characteristic of Cholesteryl 10-undecenoate. For example, the stretching frequency of the ester $C=0$ group is not observed. This suggests that the Si-OH groups on the silica surface have not been replaced by a bonded brush. 
ELEMENTAL ANALYSIS

Phase 1

The elemental analysis of Phase 1 revealed a carbon and hydrogen content of 9.69 and $0.89 \% \mathrm{wt} / \mathrm{wt}$, respectively. This carbon value corresponds to a surface coverage of 3.55 umol/M2. The above DRIFT spectrum analysis of Phase 1 suggests that this value represents a bonded brush, see figures 16 and 17 .

Phase 2

The elemental analysis of Phase 2 revealed a carbon and hydrogen content of 12.75 and $1.20 \% \mathrm{wt} / \mathrm{wt}$, respectively. This carbon value corresponds to a surface coverage of 4.74 umol/M2. The above DRIFT spectrum analysis of Phase 2 suggests that this value represents a bonded brush, see figures 18 and 19 .

Phase 3

The elemental analysis of Phase 3 revealed a carbon and hydrogen content of 1.07 and $0.23 \%$, wt/wt, respectively. From the above spectral studies, it is clear that these values do not represent a bonded brush, see figures 11, 12, 13, 14, 20 and 21. It is likely that they correspond to surface contamination of the silica by physically adsorbed aliphatic material(s); recall from figure 20 that no functionality bands characteristic of Cholesteryl 10undecenoate were present in the DRIFT spectrum of Phase 3. 


\section{THERMAL ANALYSIS}

The DSC traces of bare silica, Phases 1, 2 and 3 are shown in figures 22, 23, 24 and 25, respectively. It should be noted that the silanol groups on the silica surface decompose to siloxanes and water at approximately $350^{\circ} \mathrm{C}$.

The four DSC scans are similar with each forward trace, the upper curve, having a positive heat flow, while the reverse traces have negative heat flows. However, from 300 to $425^{\circ} \mathrm{C}$, the forward traces of Phases 1 and 2 have greater slopes relative to the forward trace of bare silica; the slopes of these traces, from 300 to $425^{\circ} \mathrm{C}$, are: $0.109,0.120$ and 0.088 for Phase 1, Phase 2 and bare silica, respectively. This suggests that an endothermic phase transition has occurred to Phases 1 and 2 within this temperature range; the exact nature of this transition is not fully understood, however, it is believed that the bonded brushes have become reoriented relative to the silica surface. A study by Claudy and coworkers [10] reported that bonded phase chain mobility increases at elevated temperatures. This increased mobility can be compared to a melting process. This could explain the endothermic transition observed in our study, for Phases 1 and 2 .

The study by Laub and coworkers quoted above [9] also employed [4-(Allyloxy)benzoyl] biphenyl and 4-[\{4-(Allyloxy) benzoyl\}oxy]-4'-methoxy biphenyl. However, in their study, the bonded phases were generated by reaction between the 
biphenyls and poly(methylsiloxane). The polymer transition temperatures reported by Laub and coworkers for the [4(Allyloxy)benzoyl] biphenyl and 4-[\{4-(Allyloxy)benzoyl\} oxy]-4'-methoxy biphenyl based polymers were $95-125^{\circ} \mathrm{C}$ and $139-319^{\circ} \mathrm{C}$, respectively. There appears to be a significant difference between the transition temperatures reported in the two studies. This should not be surprising since the solid supports used in our study differed from theirs, that is, silica vs. poly(methylsiloxane). The backbone of poly(methylsiloxane) is less rigid compared to that of silica. This rigidity could be a contributing factor causing the observed differences in the polymer transition temperatures.

Note that the forward traces of bare silica and Phase 3 are identical. This suggests that Phase 3 did not undergo any thermal phase transition and behaved as a thermal equivalent to bare silica; one would expect this from the above spectral analyses.

The reverse traces of silica and Phases 1, 2, and 3 are identical. This implies that the materials have become thermal equivalents; that is, complete decomposition has occurred to the silica and Phases 1, 2 and 3.

It is interesting to note here that while [4-(Allyloxy) benzoyl] biphenyl is not a liquid crystal, it did exhibit liquid crystalline behavior in the bonded phase, Phase 1. Therefore, there need not be the criterion that an 
organic moiety be a liquid crystal if one wishes to prepare a bonded liquid crystal stationary phase from it. A study by Sanders and Wise [11] reported that liquid crystal like behavior was obtained with a polymeric C-18 phase at subambient column temperatures. They concluded that this behavior was caused by alkyl chain rigidity and reduced chain mobility. However, bonding a non-liquid crystal organic moiety to silica does not guarantee liquid crystalline behavior of the bonded phase. Such behavior must be determined empirically, as above, by DSC and/or HPLC studies.

\section{CONCLUSION}

From the above discussions, it would appear that the method used in this study to chemically bond an organic moiety to the surface of silica may have some limitations. However, there can be no denial that this method is a viable synthetic route for the preparation of novel liquid crystal stationary phases for use in HPIC.

Euture work on phases 1 and 2 will focus upon characterizing the liquid crystallinity of these phases via HPLC. A study by Pesek and Siouffi [12] reported that a plot of $\log \left[k^{\prime}\right]$ vs. the percent of organic solvent in the eluent will be nonlinear for liquid crystal stationary phases, under reverse phase conditions. This nonlinear behavior is indicative of mesomorphic transitions occurring in the 
stationary phase. This approach will be investigated to determine if either phase exhibits such behavior. 


\section{REEERENCES}

[1]. E. Johnson and R. Stevenson, Basic Liquid Chromatography, Palo Alto, Ca., (1978), p. 1 .

[2]. D. Skoog and D. West, Principles of Instrumental Analysis, 2nd. ed., Philadelphia, Pa., (1980), pp. $670-680$.

[3] . J. Pesek and S. Swedberg, J.Chromatogr, 361, 8392, (1986).

[4]. J. Pesek and J. Graham, Anal. Chem., 49, 133-136, (1977).

[5]. J. Pesek and G. Guiochon, J.Chromatogr,, 395, 17, (1987).

[6]. J. Kohler, Chromatographia, 21, 573-580, (1986).

[7]. E.B. Priestley, "Liquid Crystal Mesophases", in Introduction to Liquid Crystals, ed. E.B. Priestley, P.J. Wojtowicz and P. Sheng, New York, N.Y., (1975), pp. 1-13.

[8]. A.L. Levine, "Structure-Property Relationships in Thermotropic Organic Liquid Crystals" in Introduction to Liquid Crystals, ed. E.B. Priestley, P.J. Wojtowicz and P. Sheng, New York, N.Y., (1975), pp. 15-18.

[9] . M.A. Apfel, H. Finkelmann, G.M. Janini, R.J. Laub, B.H. Luhmann, A. Price, W.I. Roberts, T.J. Shaw and C.A. Smith, Anal.Chem., 57, 651-658, (1985).

[10]. P. Claudy, J.M. Letoffe, C. Magot, D. Morel and J. Serpinet, I.Chromatogr, 329, 331, (1985).

[11]. I.C. Sander and S.A. Wise, Anal. Chem., 61, 17491754, (1989).

[12] . J. Pesek and A.M. Siouffi, Anal Chem, 61, 19281931, (1989). 
APPENDIX A 
Figure 1. Schematic diagrams of thermotropic liquid crystals of the (a) Smectic A order, (b) Smetic C order, (c) Cholesteric order and (d) Nematic order.

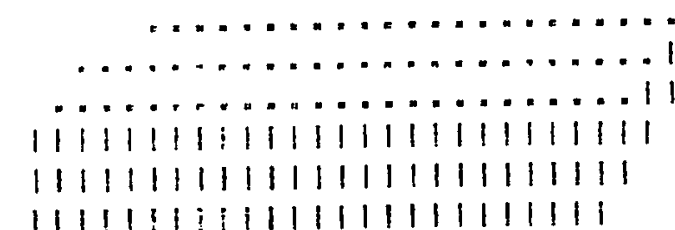

(a)

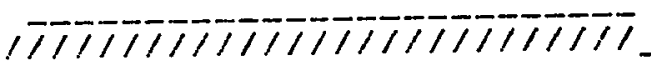
$1111111111111111111111111111^{\prime}$

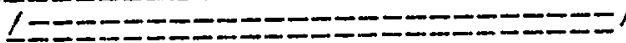

(c)

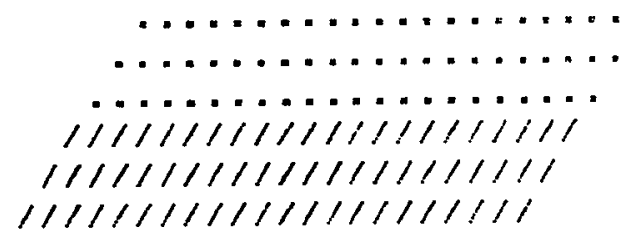

(b)

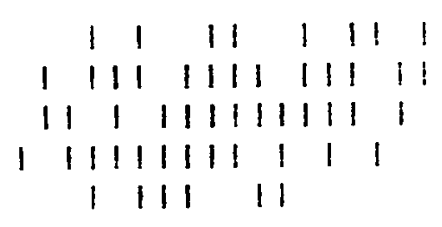

(d) 
Eigure 2. The structures of (a) [4-(Allyloxy)benzoyl] biphenyl, (b) 4-[\{4-(Allyloxy) benzoyl\}oxy $]-4$ 'methoxy biphenyl and (c) Cholesteryl 10-undecenoate.

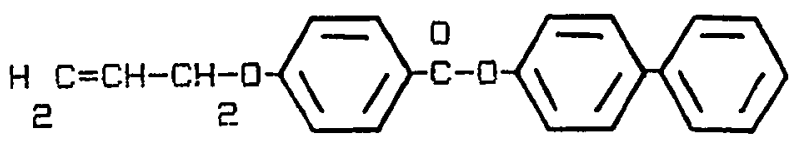

(a)

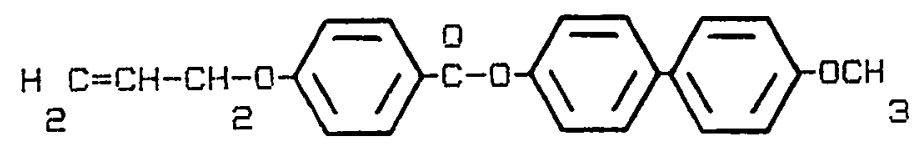

(b)<smiles>C=CC1CCCC2(C)C(=CCC3C2CCC2(C)C(C(C)CCCC(C)C)CCC32)C1</smiles>
(c) 
Figure 3. The infrared spectrum of [4-(Allyloxy) benzoyl] biphenyl.

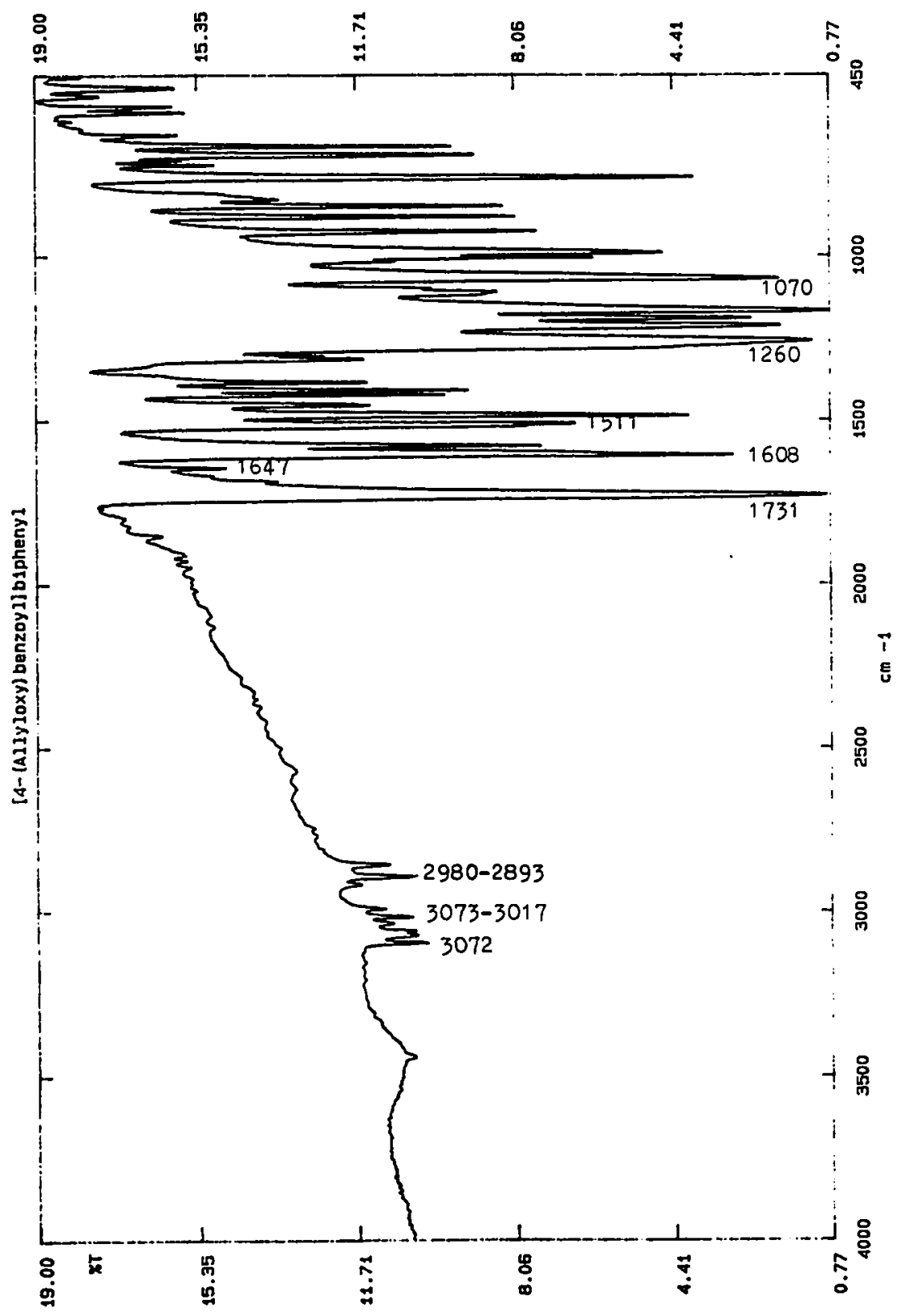


Figure 4. The H-NMR spectrum of [4-(Allyloxy)benzoyl]biphenyl.

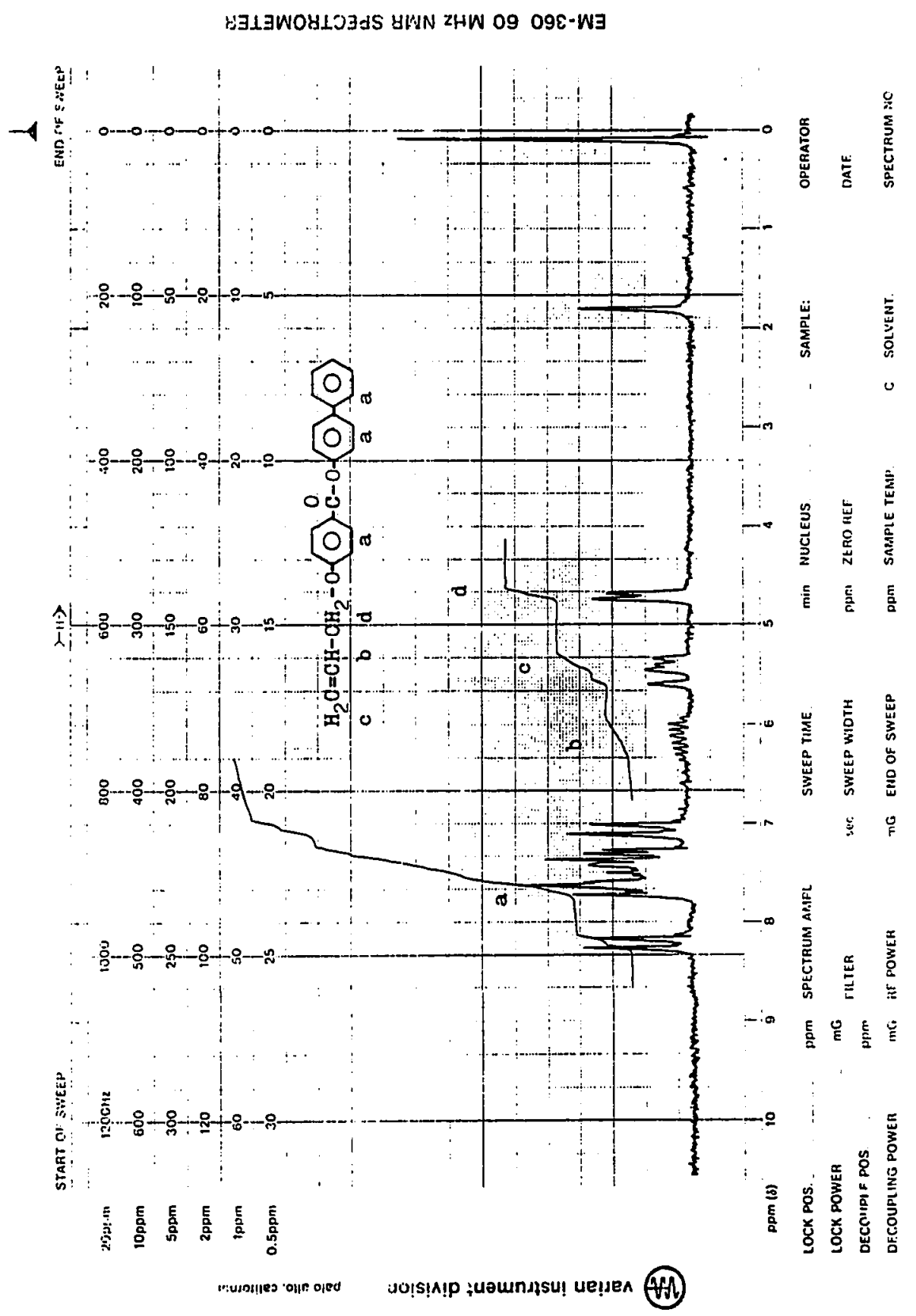

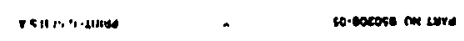


Figure 5. The infrared spectrum of [4-(Allyloxy)benzoyl] biphenyl silane reagent.

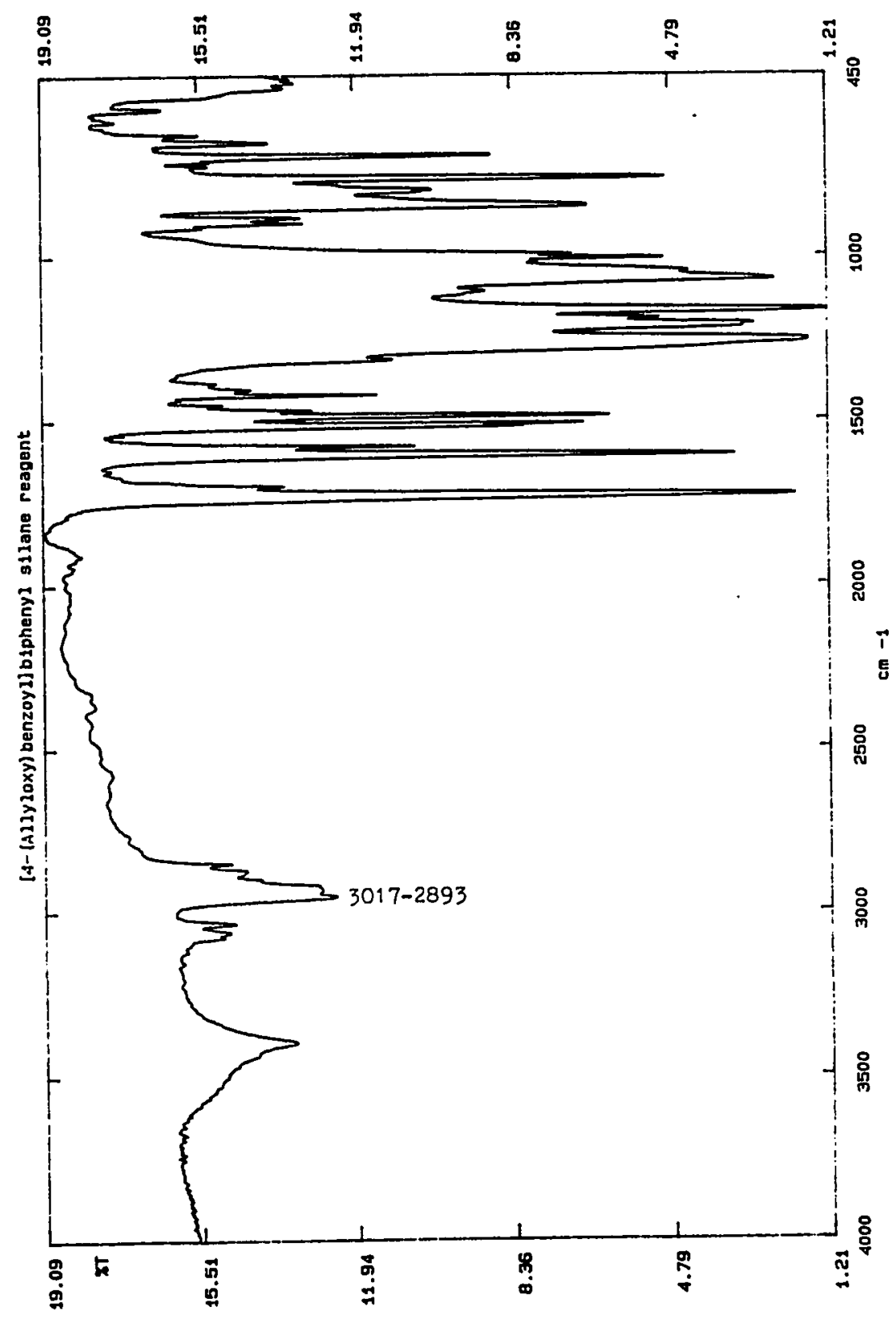


Figure 6. The H-NMR spectrum of [4-(Allyloxy)benzoyl]biphenyl silane reagent.

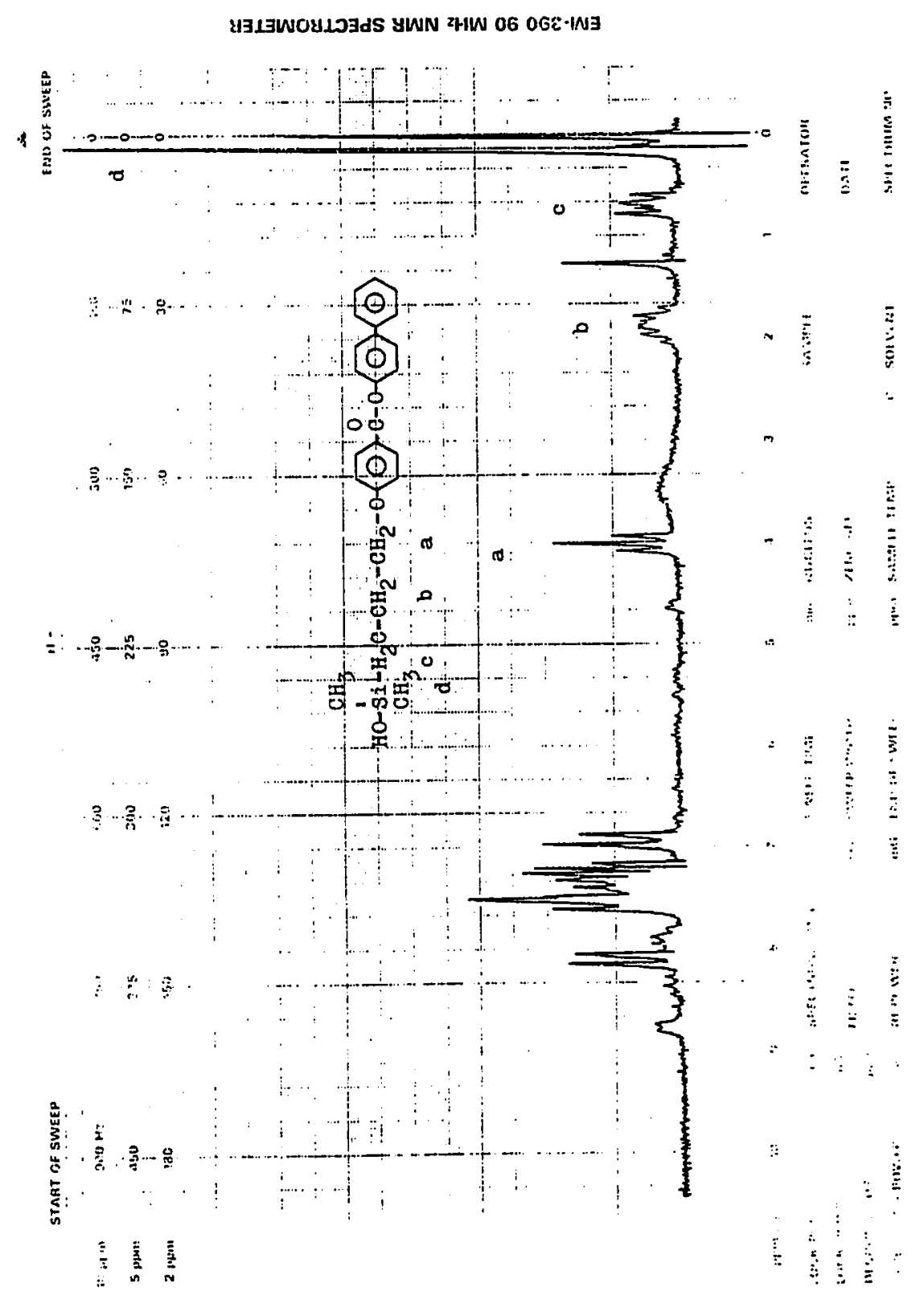


Figure 7. The infrared spectrum of $4-[(4-$ (Allyloxy) benzoyl\}oxy] -4'-methoxybiphenyl.

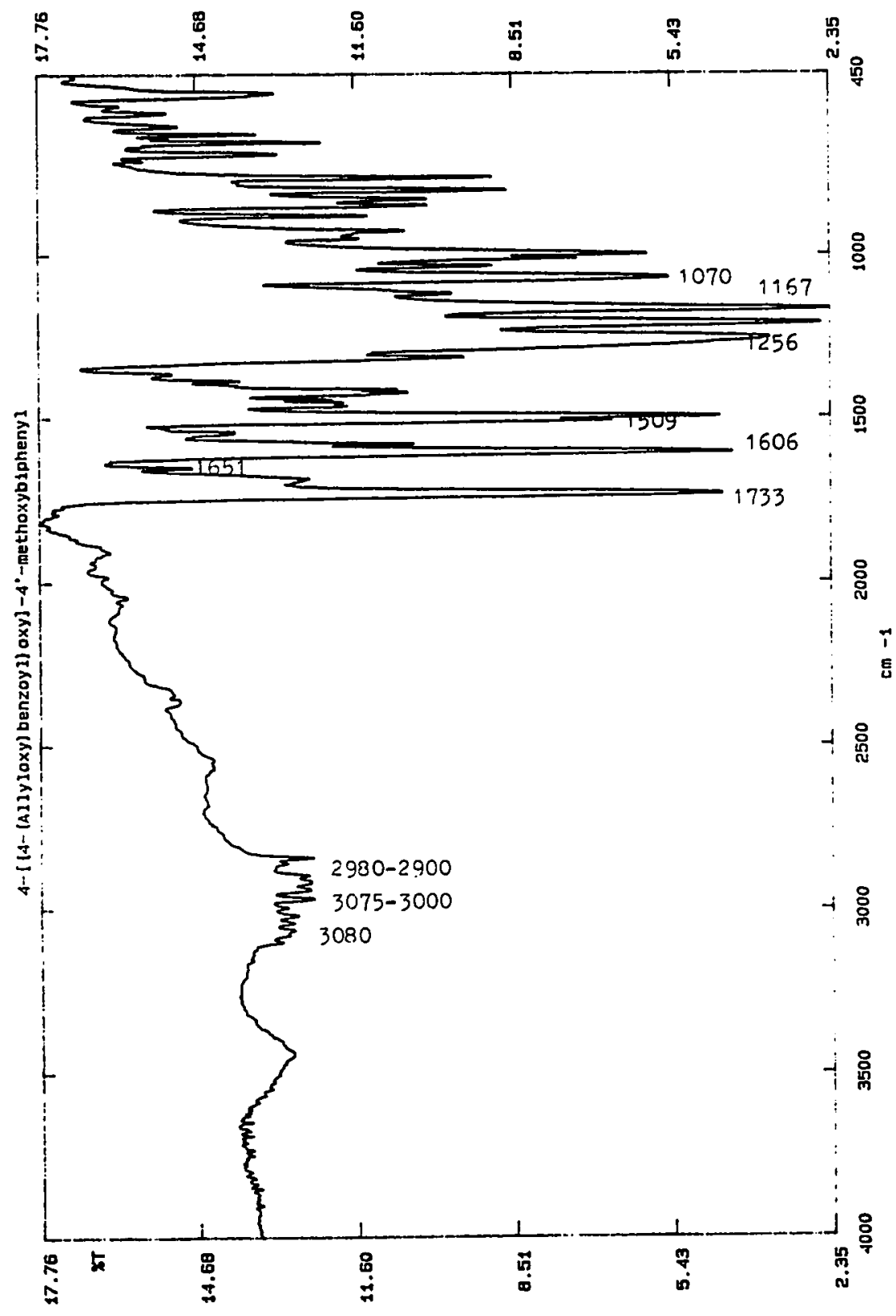


Figure 8. The H-NMR spectrum of $4-[\{4-(A 11 y$ loxy $)$ benzoyl\}oxy $]-$ 4 '-methoxybiphenyl.

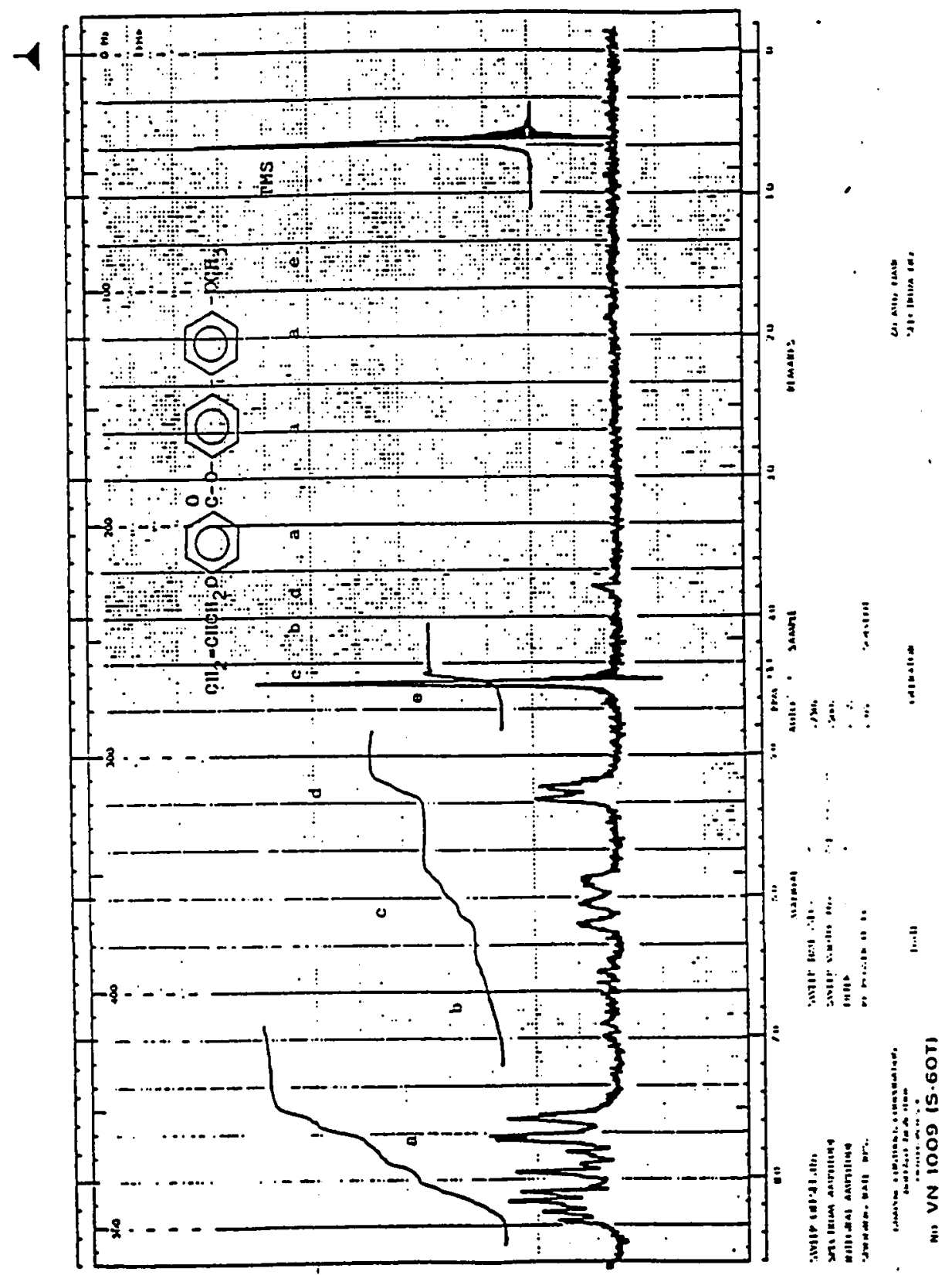


Figure 9. The infrared spectrum of 4-[\{4-(Allyloxy)benzoyl\} oxy l-4'-methoxybiphenyl silane reagent.

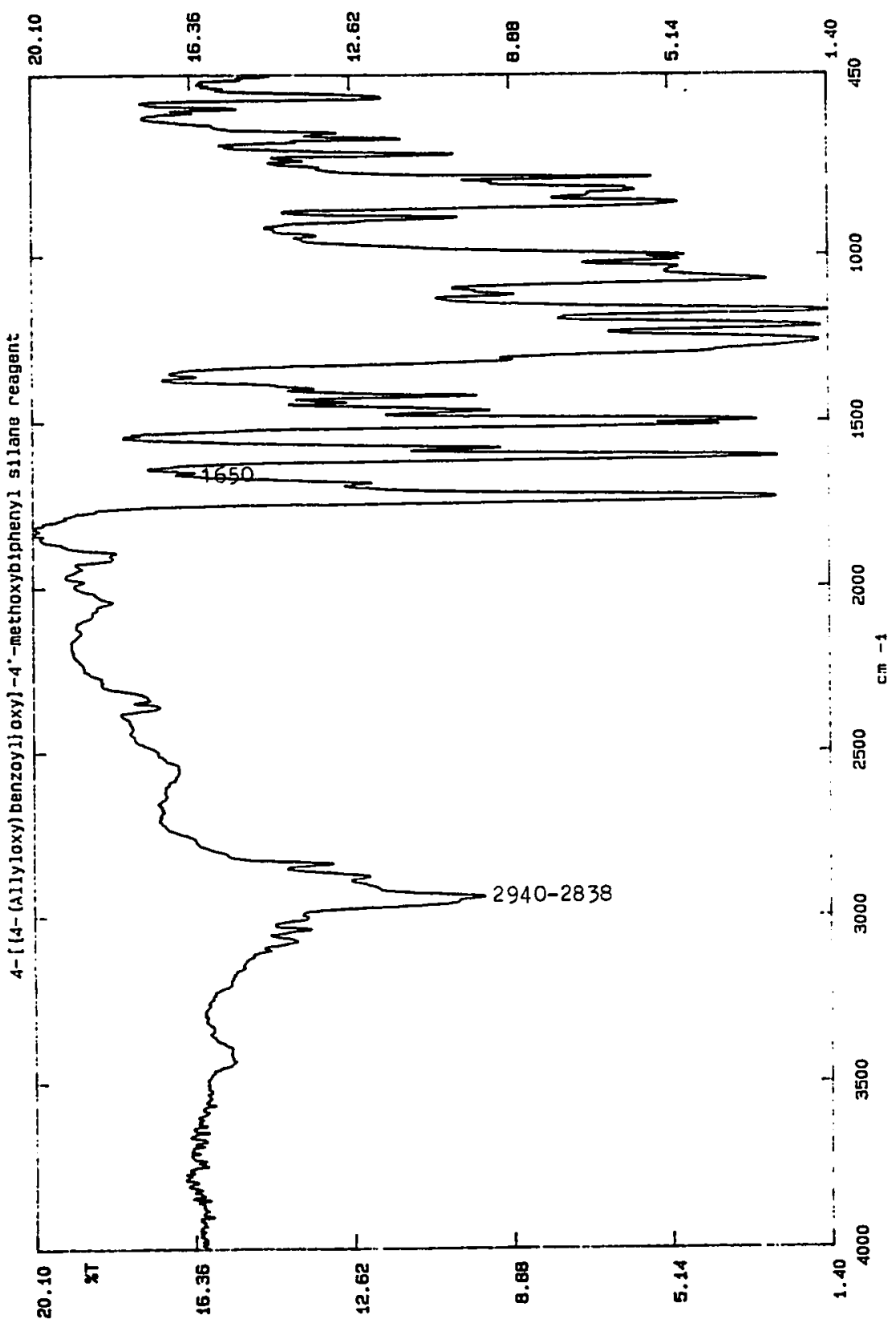


Figure 10. The H-NMR spectrum of 4-[\{4-(Allyloxy)benzoyl\}oxy]4'-methoxybiphenyl silane reagent.

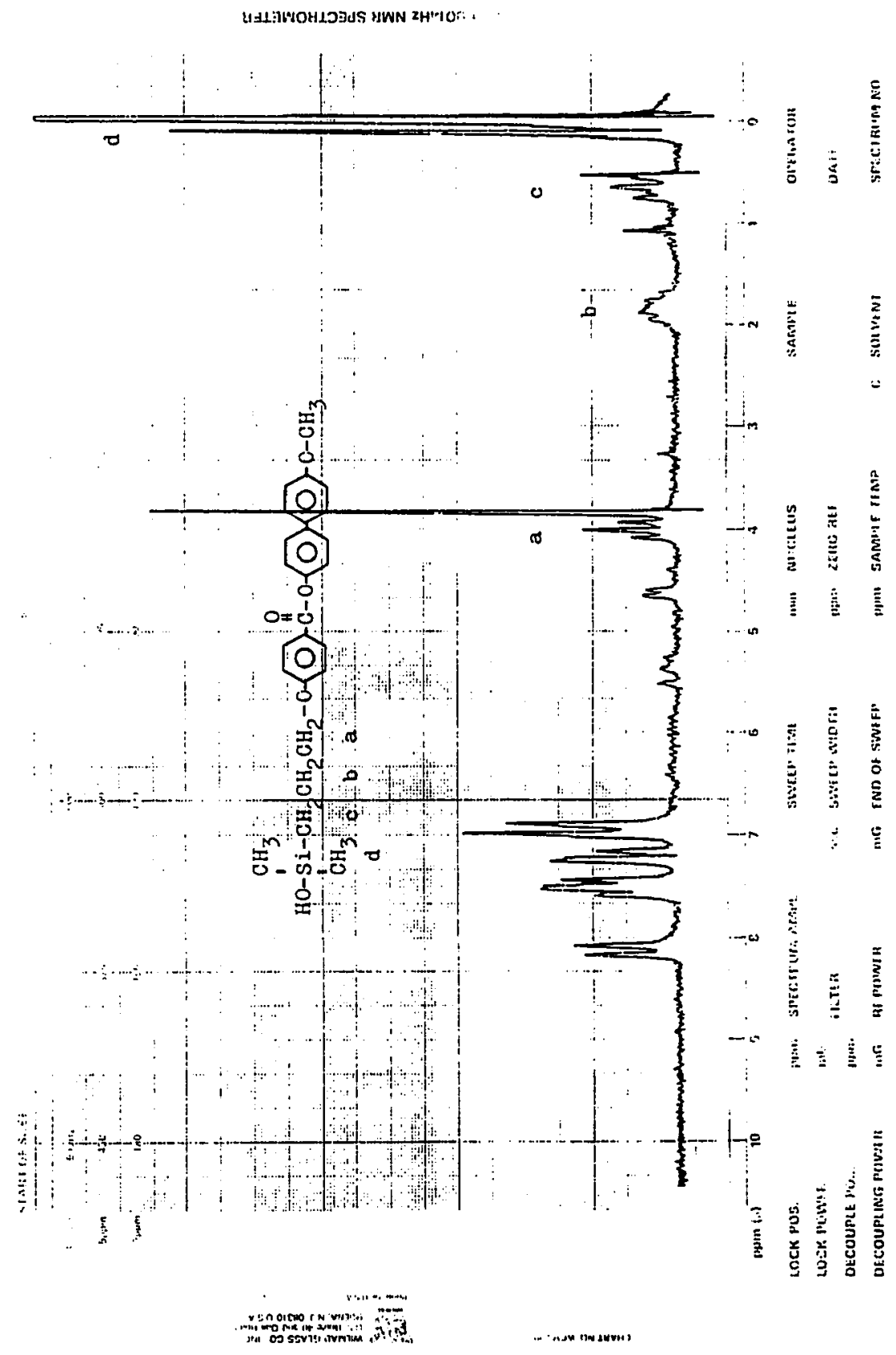


Figure 11. The infrared spectrum of Cholesteryl 10-undecenoate.

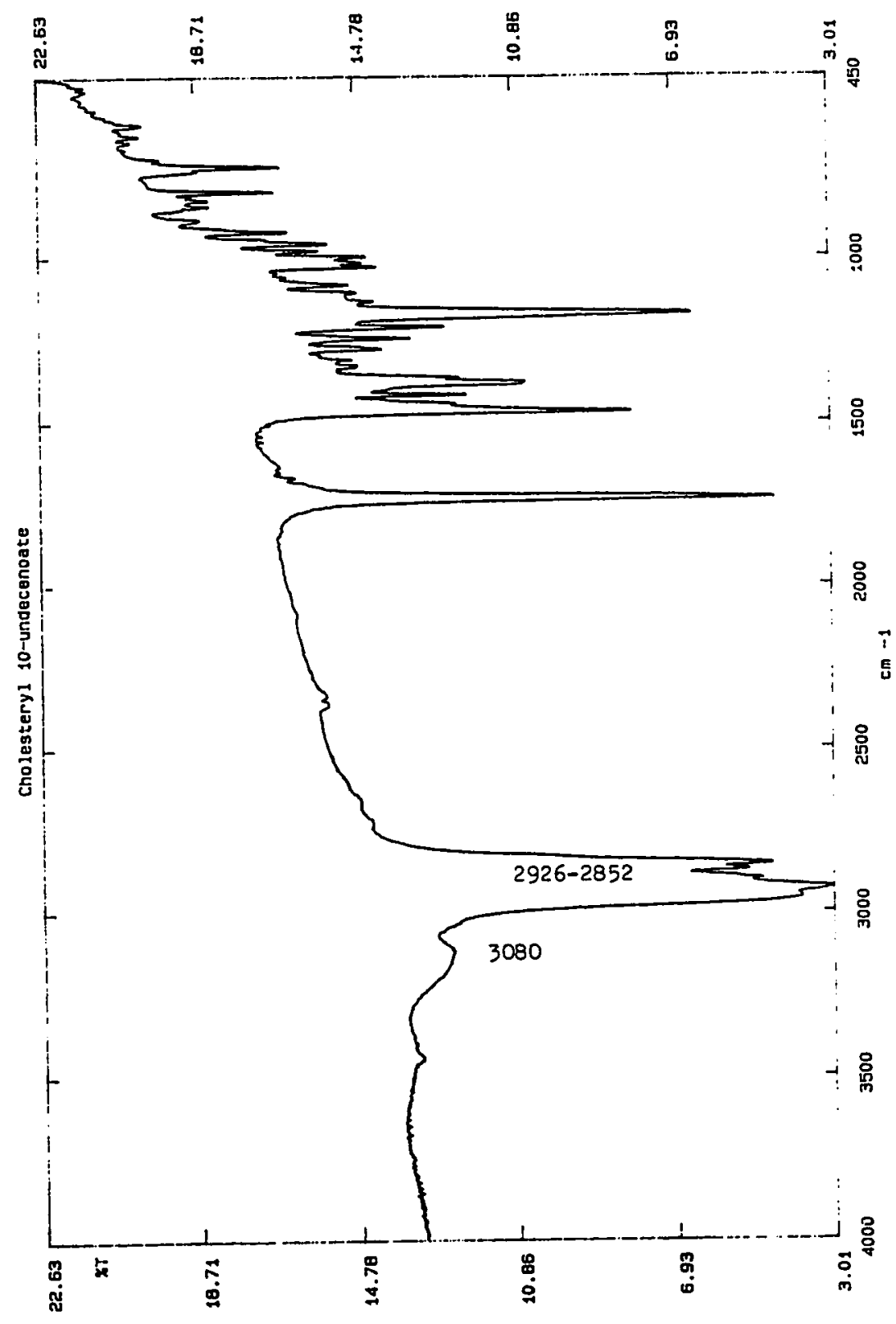


Figure 12. The H-NMR spectrum of Cholesteryl 10-undecenoate.

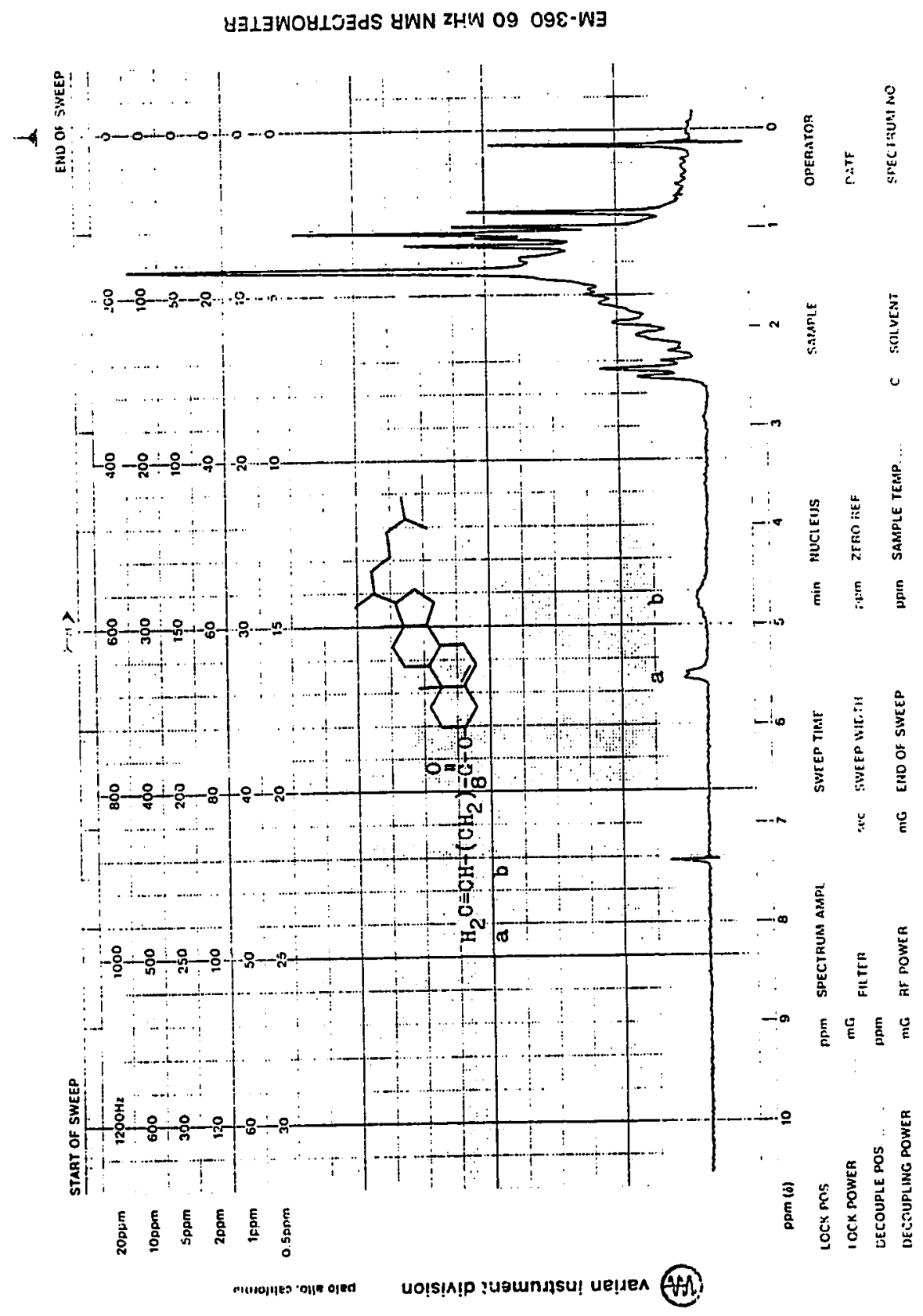


Figure 13. The infrared spectrum of Cholesteryl 10-undecenoate silane reagent.

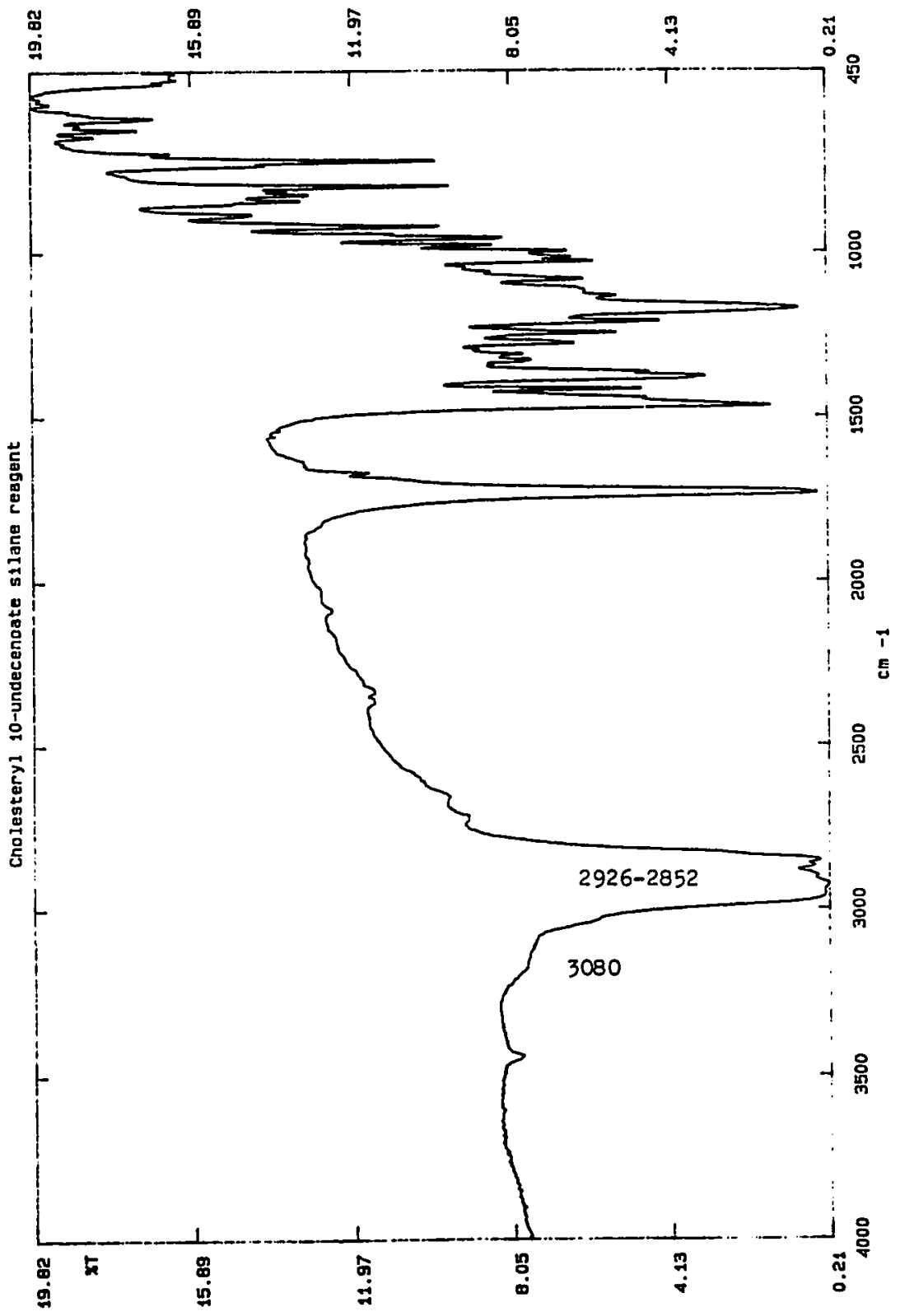


Figure 14. The H-NMR spectrum of Cholesteryl 10-undecenoate silane reagent.

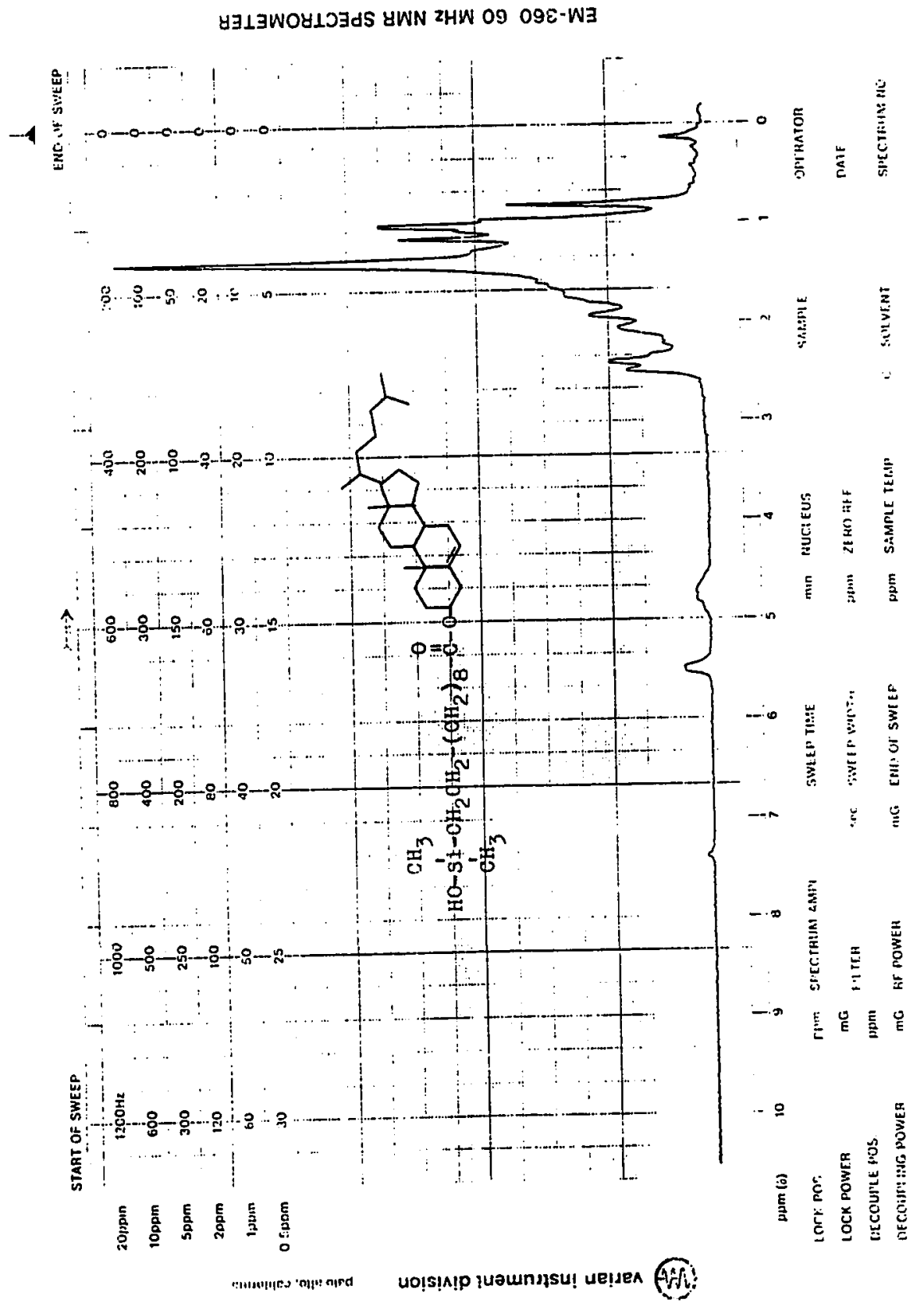


Figure 15. The DRIFT spectrum of bare silica.

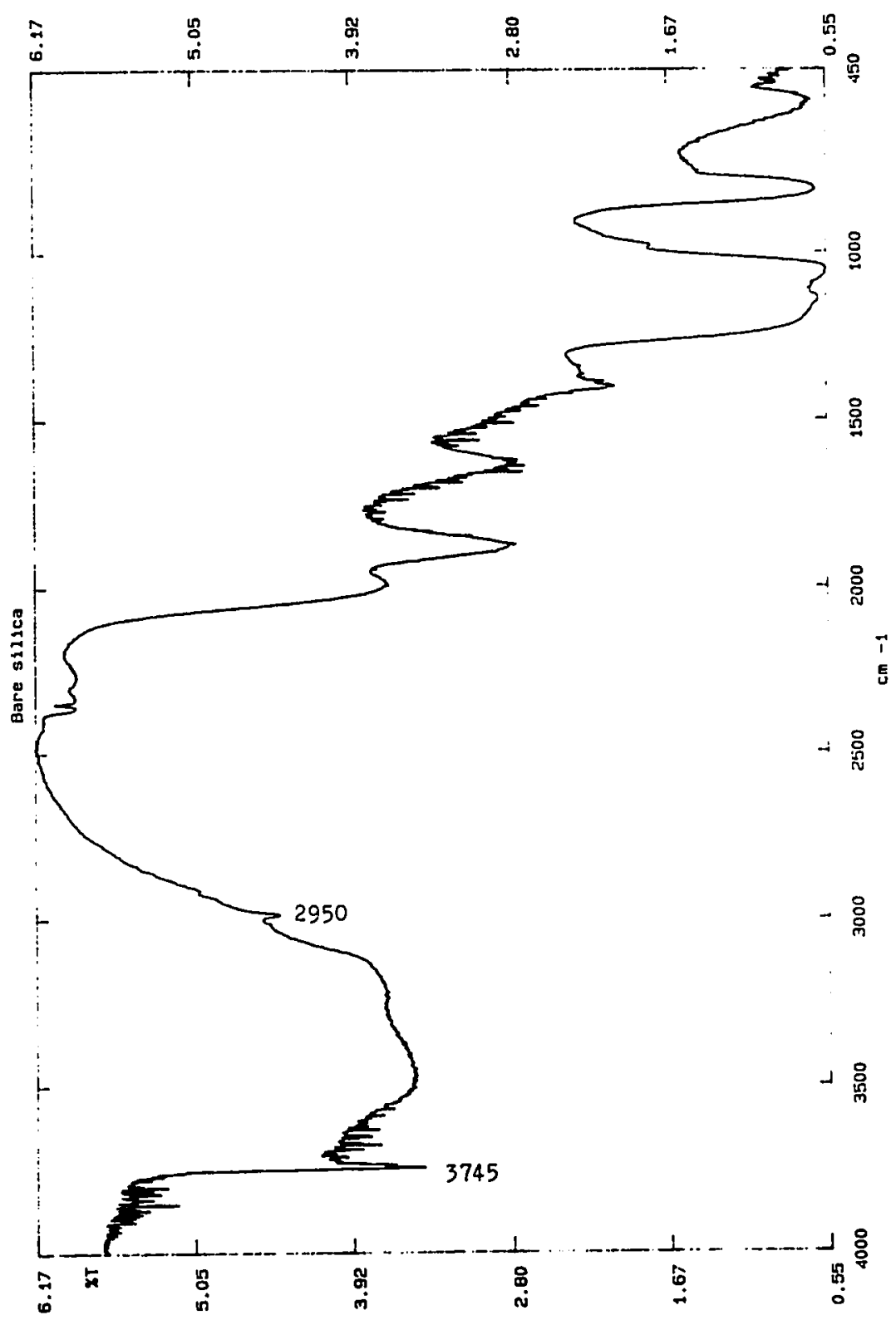


Figure 16. The DRIFT spectrum of Phase 1.

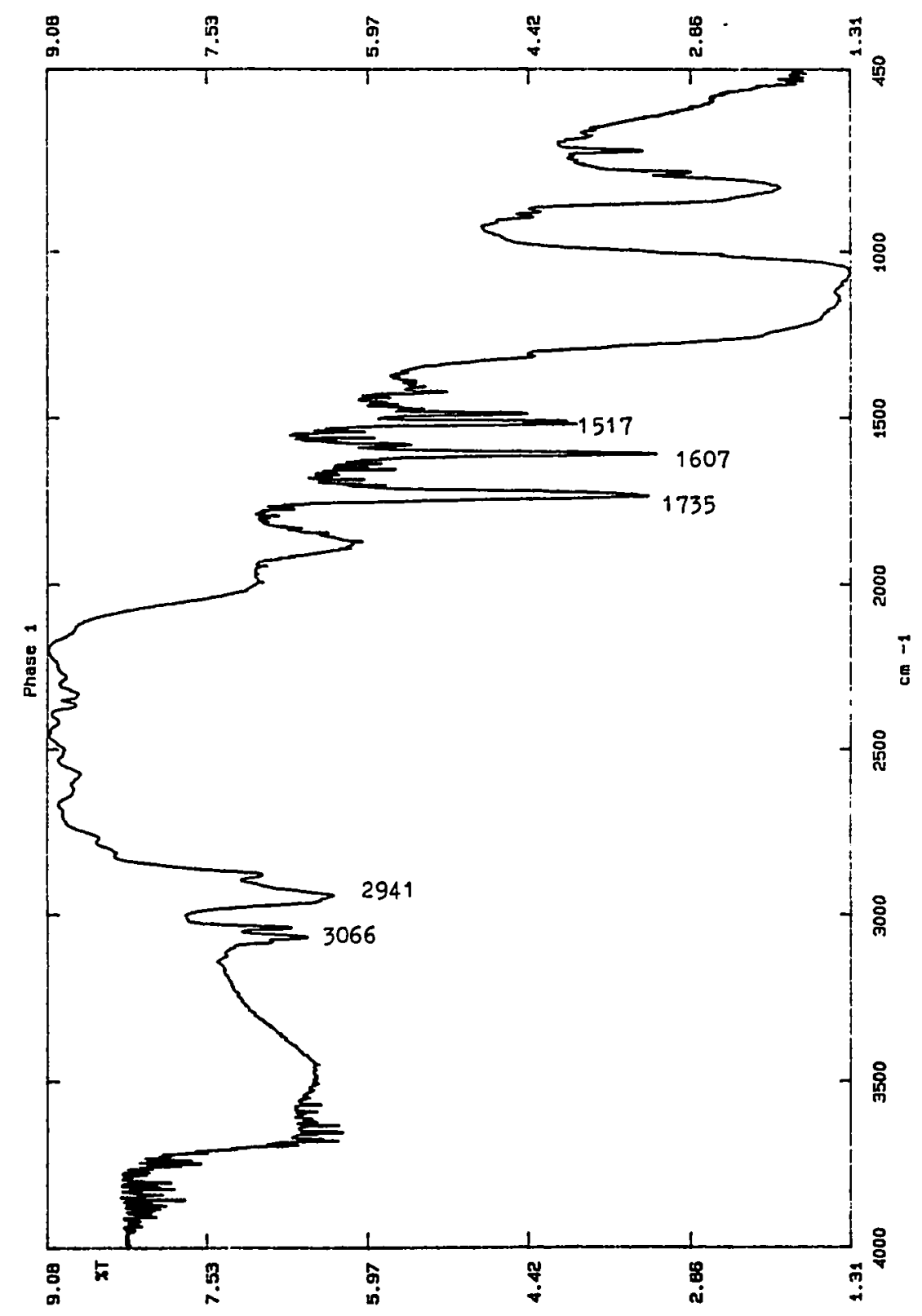


Figure 17. A comparison of the $3700 \mathrm{~cm}^{-1}$ region from the DRIFT spectra of (a) bare silica and (b) Phase 1.
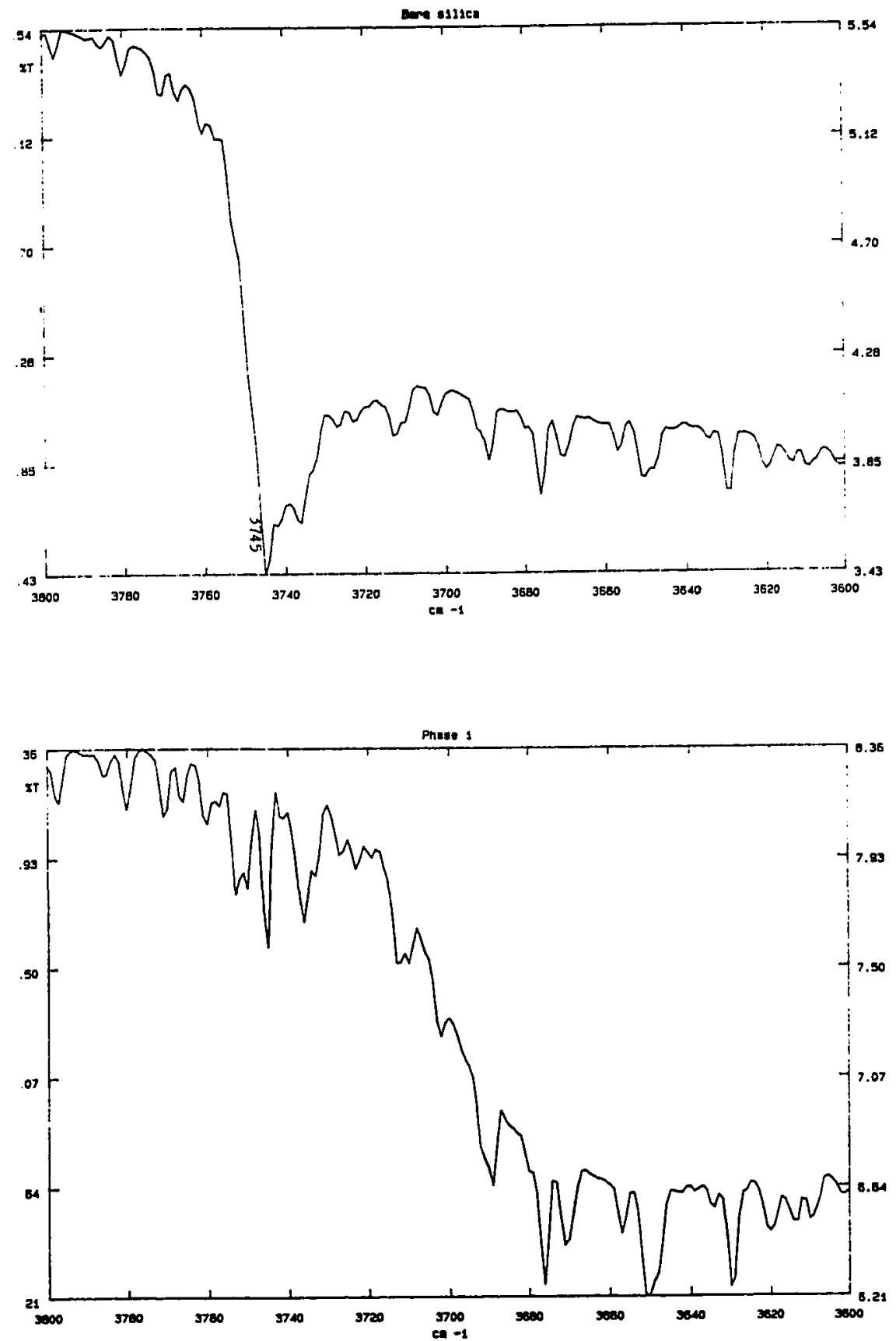
Figure 18. The DRIFT spectrum of Phase 2.

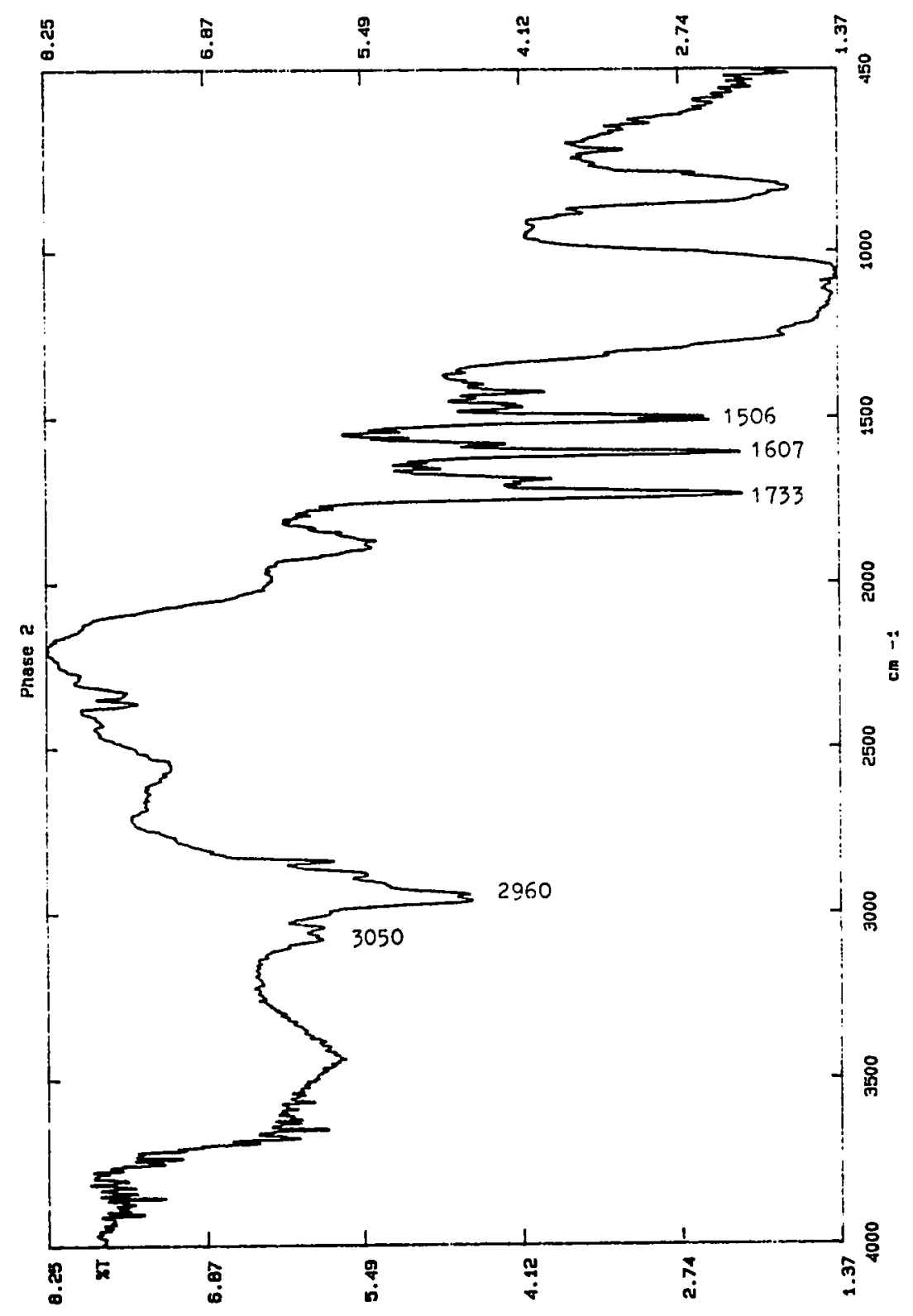


Figure 19. A comparison of the $3700 \mathrm{~cm}^{-1}$ region from the DRIFT spectra of (a) bare silica and (b) Phase 2.
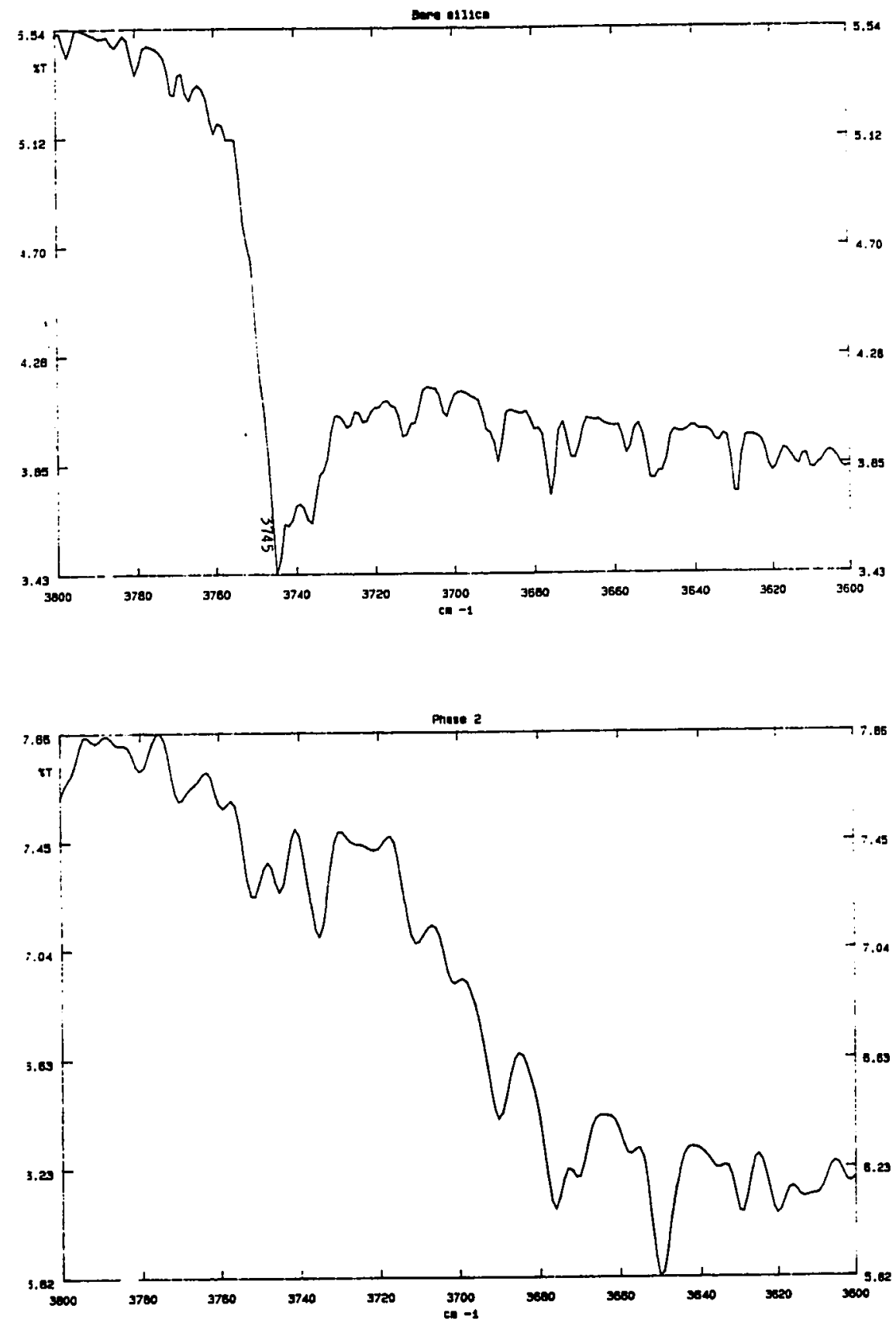
Figure 20. The DRIFT spectrum of Phase 3 .

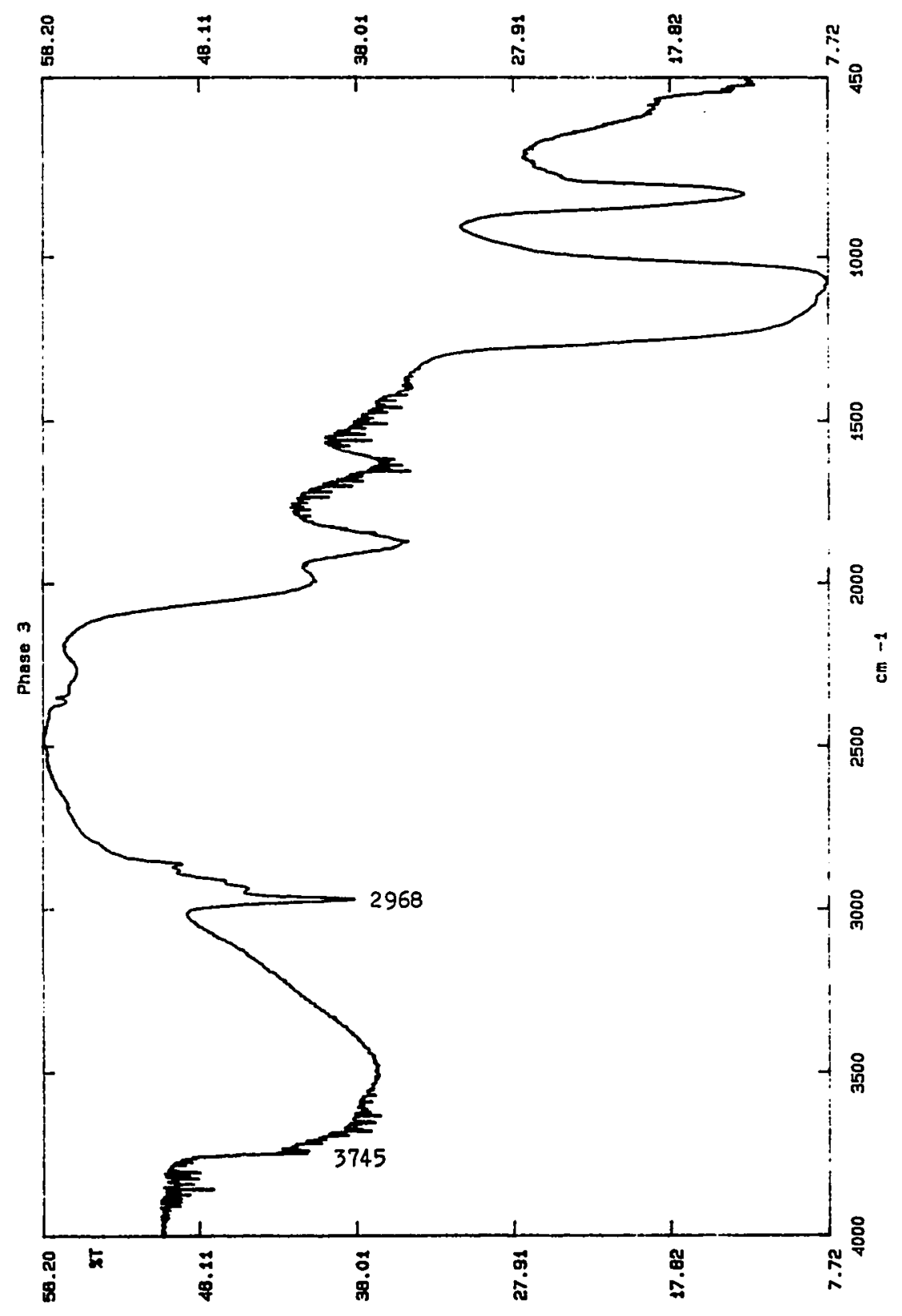


Figure 21. A comparison of the $3700 \mathrm{~cm}^{-1}$ region from the DRIFT spectra of (a) bare silica and (b) Phase 3.
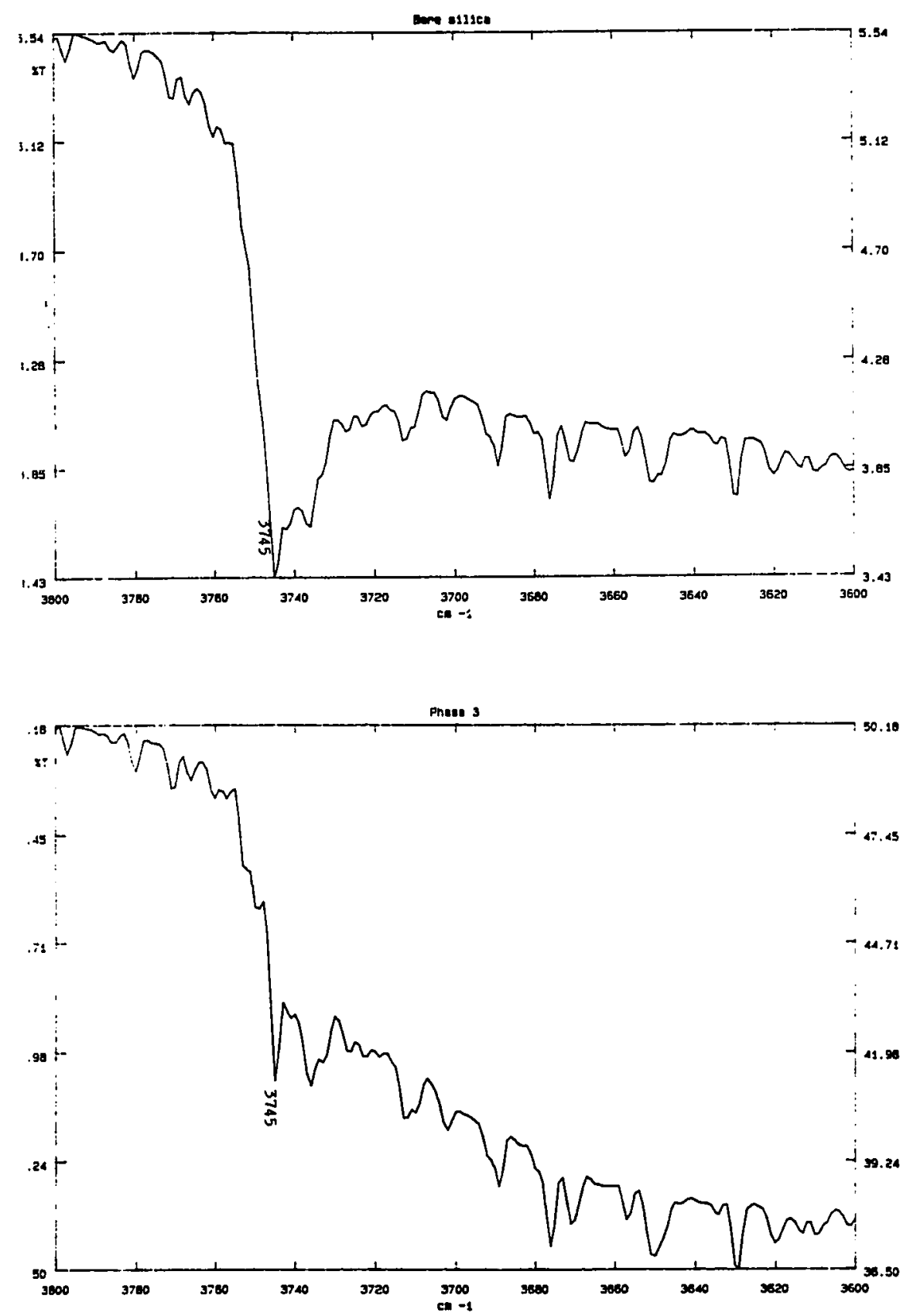
Figure 22. The DSC trace of bare silica.

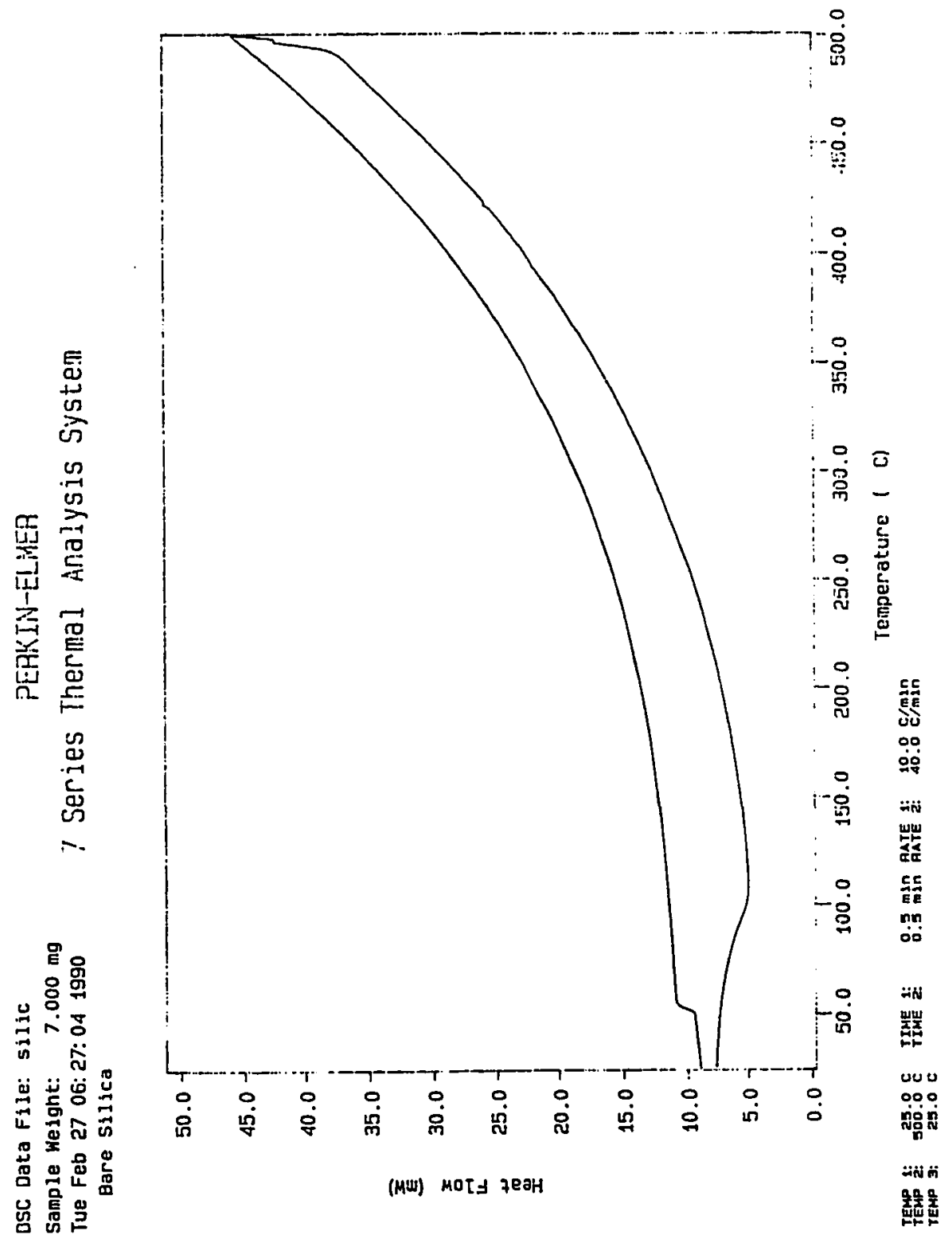


Figure 23. The DSC trace of Phase 1.

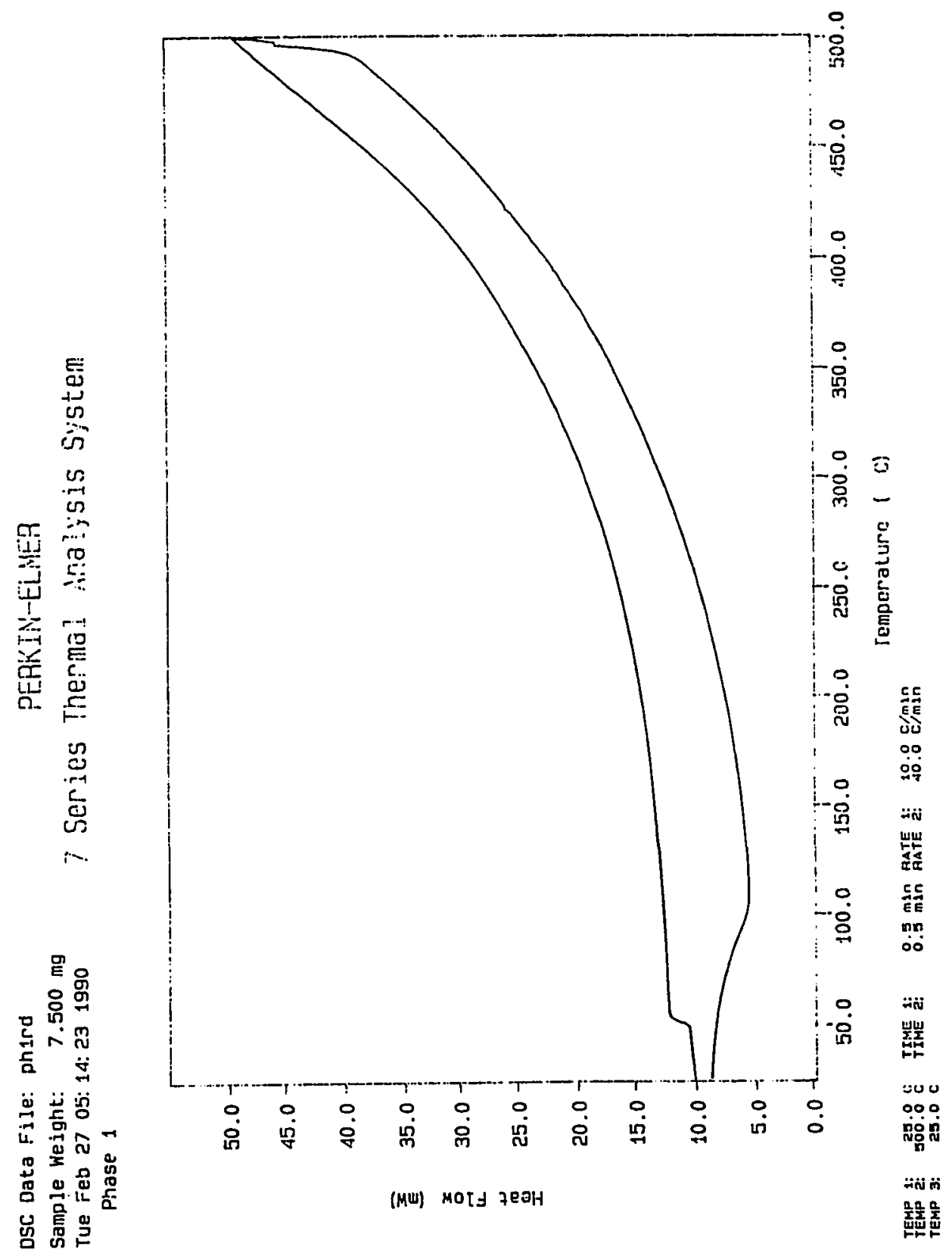


Figure 24. The DSC trace of Phase 2 .

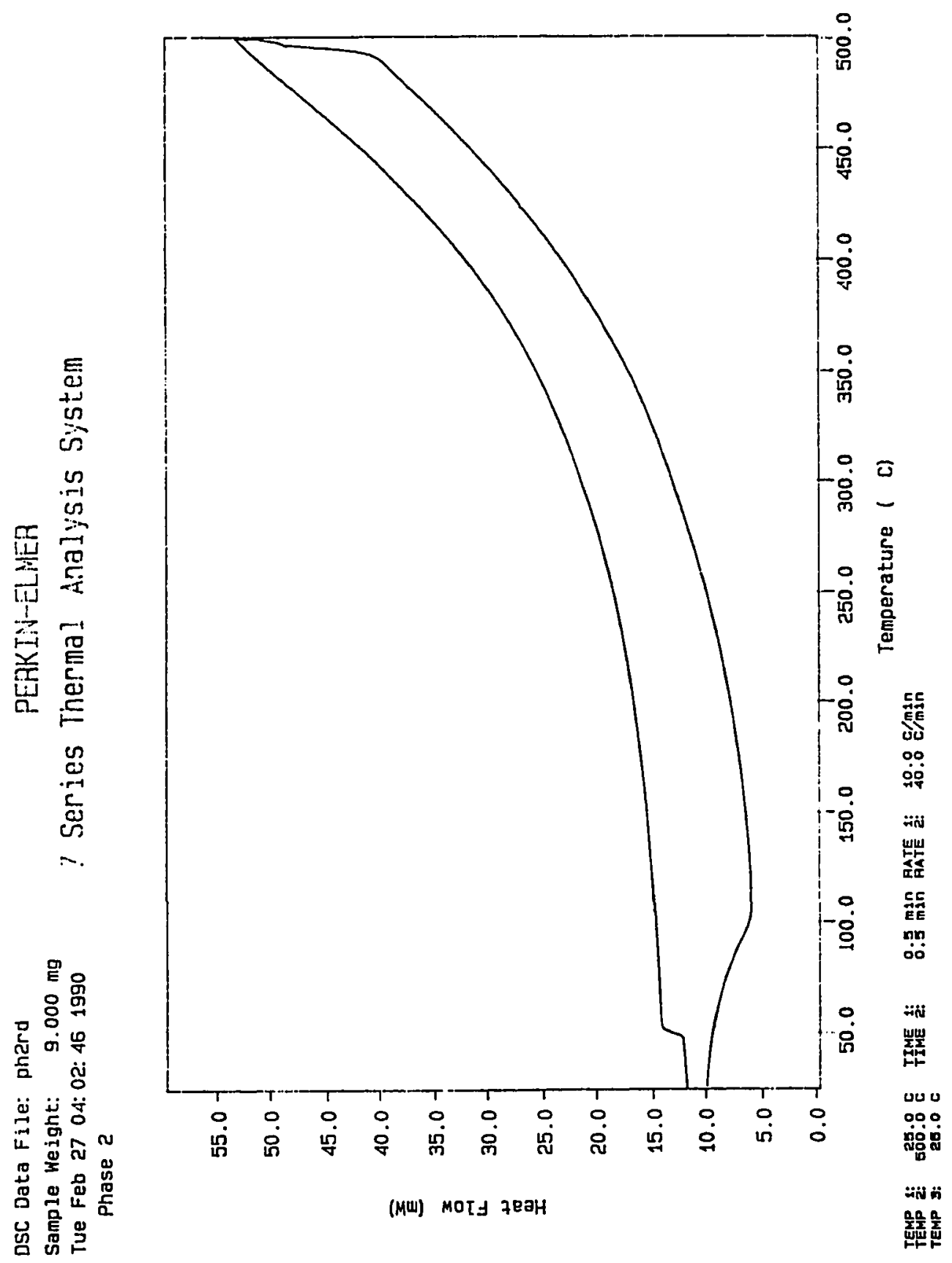


Figure 25. The DSC trace of Phase 3.

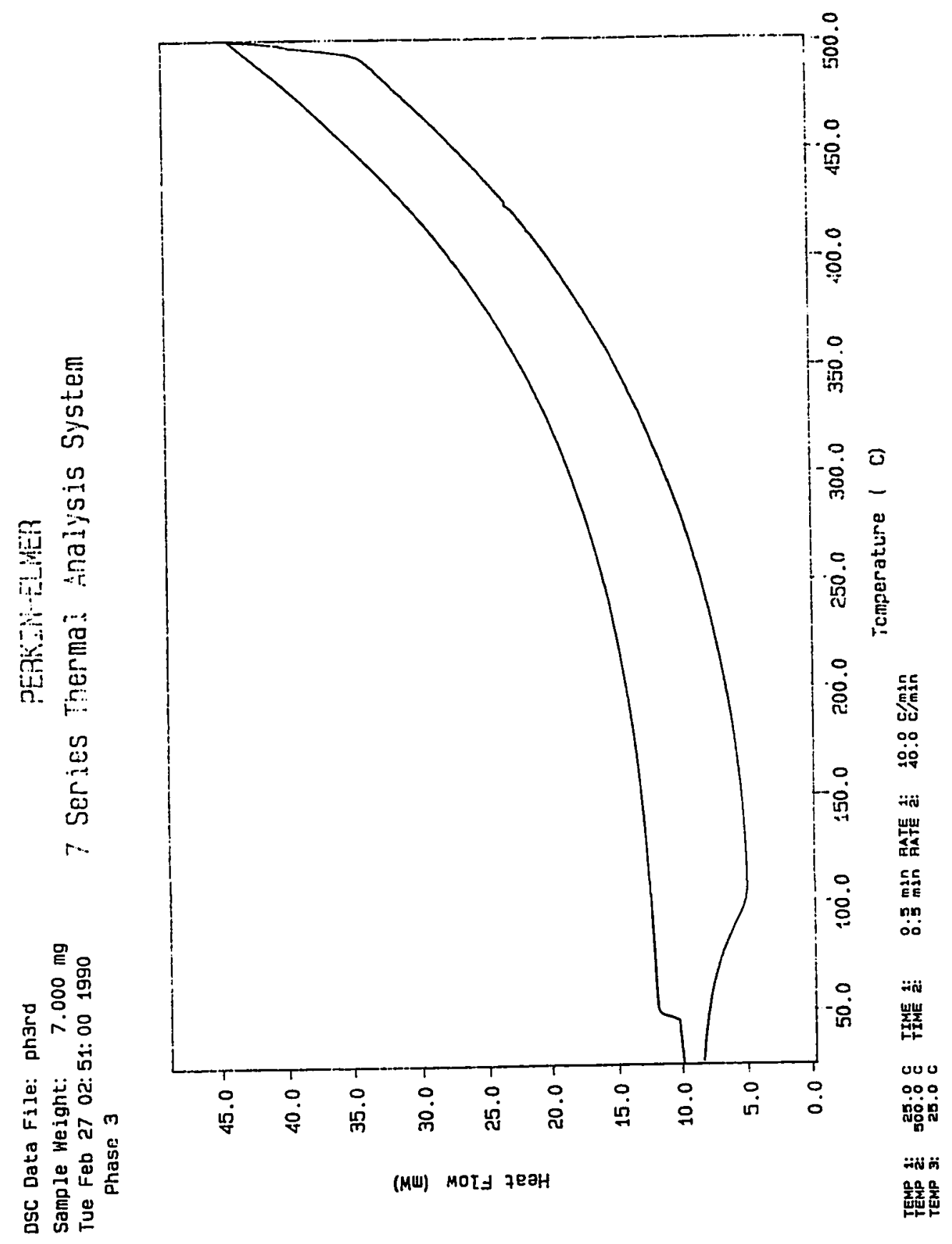

